\title{
Alleviation of Overloads in Transmission Network: A Multi-level Framework Using the Capability from Active Distribution Network
}

Jin, Xiaolong; Mu, Yunfei; Jia, Hongjie; Wu, Qiuwei; Jiang, Tao ; Wang, Mingshen; Yu, Xiandan ; Lu, Yihan

Published in:

International Journal of Electrical Power \& Energy Systems

Link to article, DOI:

10.1016/j.ijepes.2019.05.007

Publication date:

2019

Document Version

Early version, also known as pre-print

Link back to DTU Orbit

Citation (APA):

Jin, X., Mu, Y., Jia, H., Wu, Q., Jiang, T., Wang, M., Yu, X., \& Lu, Y. (2019). Alleviation of Overloads in Transmission Network: A Multi-level Framework Using the Capability from Active Distribution Network. International Journal of Electrical Power \& Energy Systems, 112, 232-251.

https://doi.org/10.1016/j.ijepes.2019.05.007

\section{General rights}

Copyright and moral rights for the publications made accessible in the public portal are retained by the authors and/or other copyright owners and it is a condition of accessing publications that users recognise and abide by the legal requirements associated with these rights.

- Users may download and print one copy of any publication from the public portal for the purpose of private study or research.

- You may not further distribute the material or use it for any profit-making activity or commercial gain

- You may freely distribute the URL identifying the publication in the public portal 


\title{
Alleviation of Overloads in Transmission Network: A Multi-level Framework Using the Capability from Active Distribution Network
}

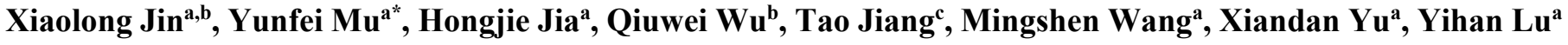

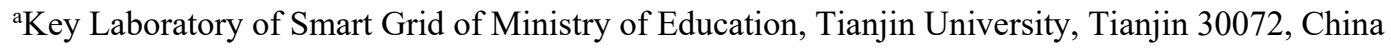 \\ ${ }^{\mathrm{b}}$ Center for Electric Power and Energy (CEE), Department of Electrical Engineering, Technical University of Denmark (DTU), \\ 2800 Kgs. Lyngby, Denmark \\ 'Department of Electrical Engineering, Northeast Electric Power University, Jilin, Jilin 132012, China \\ Email address: xljin@tju.edu.cn, yunfeimu@tju.edu.cn, hjjia@tju.edu.cn, qw@elektro.dtu.dk, tjiang@neepu.edu.cn, \\ wangmingshen@tju.edu.cn, yuxd@tju.edu.cn, luyh1996@tju.edu.cn \\ *Corresponding author: Yunfei Mu, E-mail address: yunfeimu@tju.edu.cn
}

\begin{abstract}
With the fast development of technologies of renewable generations and distributed energy resources, the distribution grids become more and more active, and the coordinated transmission and distribution management (CTDM) becomes more important. Under the CTDM framework, this paper proposes a multi-level overload relief method for transmission network using the capability from an active distribution network (ADN). When an overload event occurs at the transmission network, an appropriate operational scheme for ADN is selected by the proposed multi-level method. Then, the ADN takes the decided operational scheme and adjusts its operational status (i.e., reconfiguring its network topology, transferring loads to Microgrids and shedding the controllable loads) to contribute to load curtailment from the ADN side and further alleviate overload in the transmission network. Numerical case studies show that the proposed method can alleviate the overload of transmission network and maintain the normal operation of the $\mathrm{ADN}$, which demonstrates a promising application for CTDM.
\end{abstract}

Keywords: Coordinated transmission and distribution management, Alleviation of overload in transmission network, Active distribution network, Multi-level framework

\section{INTRODUCTION}

\section{A. Background}

Driven by the low carbon economy to increase the penetration of distributed energy resources (DERs) in recent years, the traditional 'passive' distribution network becomes more and more 'active'. Active distribution network (ADN) has drawn more and more attention due to its capability of coordinating the DERs (i.e., distributed generators and energy storages) and flexible 
loads [1]. With this capability, the ADN can: 1) optimize its operational schedules together with the transmission system under normal states of the transmission system for economic and environmental purposes; 2) adjust its operational schedules to help the transmission system with the operational issues (e.g., overload) under emergency states of the transmission system. In this context, it leads to the concept of coordinated transmission and distribution management (CTDM) [2].

\section{B. Motivation}

Several benefits can be achieved by applying the CTDM on transmission system and ADN: 1) cost-effectiveness of both the transmission system and ADN can be realized [3];2) large power mismatch at the boundary buses between the transmission system and ADN can be avoided [4]; 3) intermittent renewable generation can be more efficiently integrated at both transmission level and distribution level [5]; 4) the operational risk of the transmission system can be reduced using the CTDM method [6]; 5) the transmission system and ADN can be designed and planned with less investment using CTDM-based planning method [7]. Therefore, CTDM has become a hot topic due to its benefits for modern power systems.

\section{Related work and research gaps}

\section{1) CTDM}

Several achievements have already been made regarding the CTDM, which are categorized into two main streams according to the operational states of the transmission system [2]-[9].

In the first category, the transmission system and the ADNs are operated together in a secure and economic manner under normal states of the transmission system. A global power flow method was proposed in [2] to achieve a global unified power flow analysis for integrated transmission and distribution systems. A unit commitment method was proposed for integrated transmission and distribution systems in [3] using a decentralized decision-making framework. The economic dispatch methods of transmission system and ADN were proposed in [4]-[5] to schedule the generation resources and evaluate the locational marginal prices of the integrated system in an optimal way. A hierarchical method was proposed in [6] to evaluate the impact of role changing of the distribution network from 'passive' to 'active' in risk assessment of transmission network under the CTDM framework.

In the second category, the ADNs are operated to help the transmission system with the operational issues under emergency states (e.g., overload, voltage fluctuations) of the transmission system. Transmission contingency analysis methods were proposed in [8]-[9] considering the CTDM. It is suggested that global power flow-based transmission contingency analysis should be performed instead of only transmission power flow based contingency analysis to avoid potential false alarms. Ref. [10] proposed a CTDM method, in which the parallel transformers owned by the ADNs are controlled to generate reactive power and provide 
voltage support for the transmission networks.

The above research works have made significant contributions for CTDM under both normal states and emergency states of the transmission system. However, there is still a lack of operating method for ADNs aiming to help the transmission system alleviate its overload. Actually, the operational decisions of the transmission networks and ADNs can be made with the information exchange between them under the CTDM framework [11]. In this context, the flexible capability of ADNs can be used to help alleviate the overload in transmission networks.

\section{2) Approaches of overload relief in transmission network}

The traditional approaches to alleviate overload in transmission network mainly lies in the following three aspects [12]:

- Transmission network reinforcement

Reasonable reinforcement is important for alleviating overload in transmission network. A multi-objective transmission expansion planning method was developed in [13] to reinforce the transmission network and alleviate its overloads. Flexible AC Transmission Systems (FACTS) devices were used in [14]-[15] to reinforce the transmission network, which can remove the overloading effect.

- Generation rescheduling

Generation rescheduling is a practical method for overload relief in transmission network. A directional control method for interface flow by rescheduling the generators based on surface approximation was proposed in [16] to alleviate overload in transmission network. A multi-objective based generation rescheduling method was proposed in [17] to alleviate overload in transmission network. In [18], an overload relief strategy for transmission network was proposed based on a fuzzy adaptive bacterial foraging algorithm by optimal rescheduling the participating generators. An overload emergency state control method was proposed in [19] to alleviate overload in transmission network.

- Load shedding

The load shedding is usually used as a control measure to help the power system alleviate its overload [20]. In [21], a load shedding algorithm was proposed for alleviating overload in transmission lines by employing the teaching learning-based optimization, which determines the optimal load shedding at selected buses based on the sensitivity of severity index. A load shedding strategy for overload relief in transmission network was proposed in [22], which involves real-time dynamic thermal line rating technology into the conventional load shedding strategies.

The above research works have made significant contributions to overload relief in transmission network. However, these approaches have mainly focused on transmission networks themselves only, while fail to consider the capability from the ADN. To 
bridge the research gaps according to the above literature review (i.e., CTDM methods [2]-[10] and traditional approaches for overload relief in transmission network [13]-[22]), a multi-level hierarchical structure is adopted and conducted on the ADN to alleviate overload in transmission network under its emergency states.

\section{Contributions of this paper}

The main contributions are summarized as follows:

1) An overload relief method in the transmission network is proposed in this paper, which includes an active reconfiguration scheme, a load transferring scheme and a demand response scheme. When an overload event occurs at the transmission network, a proper operational scheme for ADN is selected from the multi-level schemes using the proposed method. Then, the ADN adjusts its operational status by reconfiguring its network topology, transferring loads to Microgrids and shedding the controllable loads to contribute to load curtailment from the ADN side and further alleviate overload in the transmission network.

2) Compared with the traditional approaches to alleviate overload in transmission network, the proposed multi-level method not only can capitalize on the capability from the $\mathrm{ADN}$, but also can promote the interaction between the transmission network and ADN under the CTDM framework.

The structure of this paper is summarized as follows. In Section II, the framework of the proposed multi-level method is presented. Section III presents a detailed description and the mathematical model of the proposed multi-level method, including the formulations of the active reconfiguration scheme, the load transferring scheme and the demand response scheme, and their solving algorithms. Section IV discusses the operational results of the ADN under different overload events of the transmission network and demonstrates the effectiveness of the proposed method by carrying out several scenarios. Finally, Section $\mathrm{V}$ concludes the whole paper.

\section{FRAMEWORK OF THE MULTI-LEVEL METHOD}

\section{A. Capability of ADN for overload relief in transmission network}

A Microgrid (MG) normally consists of a collection of loads and DERs in a local area, which provides an effective way to handle the variability and uncertainty of the DERs [23]. MGs can be coordinated by the ADN in grid-connected mode, and can also operate in islanded mode supplying reliable electricity to local areas [24]. The MG control center (MGCC) can manage its DERs and controllable loads in an optimal way [12]. Also, it coordinates with the upstream active distribution management system (ADMS) to adjust the power output from the MG in a coordinated way. At the demand side, the increasing number of EVs can help to reduce the peak load level of the ADN by controlling their charging processes effectively [25]. Distribution network with high 
penetration of DERs and controllable loads, e.g., EVs, becomes more and more 'active', which shows the capability of supporting secure operation of the transmission system under the CTDM framework. The controllable power output of DERs are included into the MGs in this paper. And the controllable power output of the MGs is used in the proposed method to help alleviate the overload in the transmission network. Therefore, the flexible capacity of RESs in the scope of the MGs is also considered in this paper.

\section{B. Framework of the multi-level method}

The proposed method, which utilizes the capability from ADN to alleviate overload in transmission network, is illustrated in Fig. 1. When overload event occurs and further leads to the emergency state of the transmission network, the power system dispatching center (PSDC) detects the overloaded transmission lines and calculates the required load curtailment by running the power flow analysis under its current state [6]. The power flow analysis program is one of the functions of the PSDC and it can be called to calculate the required load curtailment for overload relief [26]. Then, the PSDC sends overload relief request and its required load curtailment to the ADMS.

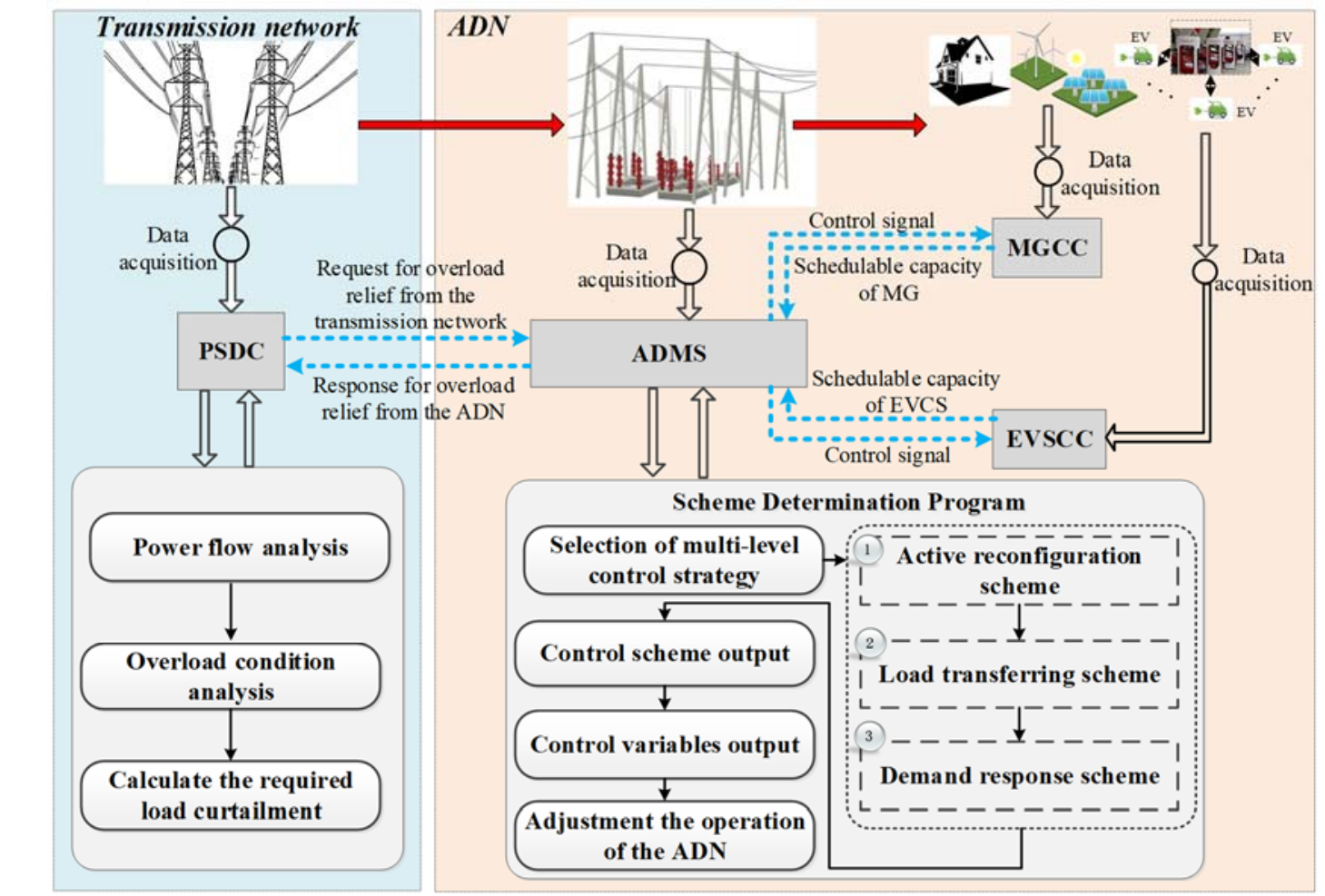

-- - : Control flow $\Longrightarrow$ : Power flow $\Longleftrightarrow$ : Data acquisition PSDC : Power system dispatching center ADMS: Active distribution managem ent system MGCC: MG control center EVSCC: EV charging station control center ADN : Active distribution network EVCS: EV charging station

Fig. 1. Framework of the multi-level method.

After that, the ADMS chooses an appropriate operational scheme for the ADN to contribute to load curtailment from the ADN side by running the scheme determination programming, as shown in Fig. 1. The load curtailment from the ADN helps to alleviate the overload of the transmission network, which is how the capability from ADN used to alleviate the overload in transmission 
network. The scheme determination program is run based on information including 1) the required load curtailment from PSDC;2) the information exchange between the ADMS and the MGCC; 3) the information exchange between the ADMS and the EVSCC.

Then, the ADMS takes the determined operational scheme and applies it through the coordination among the ADMS, MGCC and EVSCC, which is implemented following several rules. Rule 1: When the active reconfiguration scheme is determined, the ADMS adjusts the topology of the ADN by running the network reconfiguration optimization to reduce its power loss; Rule 2: When the load transferring scheme is determined, the ADMS transfers proper capacity of loads to the MGs, and schedules the power output of the MGs to accept the transferred loads; Rule 3: When the demand response scheme is determined, the ADMS schedules the power output of the MGs and the charging load of EV charging station (EVCS) to transfer larger capacity of loads to MGs on the basis of load transferring scheme. The detailed process of implementing the determined operational scheme is introduced in Section III. The MGs are coordinated by the upstream ADN rather than owned by the ADN. They are different entities and operated in a coordinated way to help alleviate the overload in the transmission network with the proposed method in this paper.

After implementing the determined operational scheme, the ADMS calculates the load curtailment from ADN side by running the power flow analysis of the ADN. Then, the ADMS sends the load curtailment to the PSDC to respond to its overload relief request. The multi-level strategy has three layers:

1) The active reconfiguration scheme is conducted at the first layer. The ADMS adjusts the topology of the ADN in an optimal way to reduce its power loss. The reduced power loss of the ADN is contributed as load curtailment from the ADN side in the active reconfiguration scheme.

2) The load transferring scheme is conducted at the second layer. The ADMS schedules the power output of the MGs to accept the transferred loads from the ADN. The transferred loads from the ADN to the MGs are contributed as load curtailment from the ADN side in the load transferring scheme.

3) The demand response scheme is conducted at the third layer. The ADMS schedules the power output of the MGs and the charging load of EVCS to transfer larger capacity of loads to MGs on the basis of load transferring scheme. The total capacity of transferred loads from the ADN to the MGs are contributed as load curtailment from the ADN side in the demand response scheme. The load curtailment from the ADN helps to alleviate the overload of the transmission network.

It is worth noting that all the information exchange (i.e., coordination between the PSDC and the ADMS, coordination between the ADMS and the MGCC, coordination between the ADMS and the EVSCC) are realized thanks to the communication, monitoring and control infrastructure of the transmission network and the ADN. 


\section{FORMULATION OF THE MULTI-LEVEL METHOD}

The multi-level method is formulated in this section. The flow chart of the multi-level method is depicted in Fig. 2. As shown in the left-side part of Fig. 2, when overload event occurs at the transmission network, the PSDC detects the overloaded transmission lines and calculates the required load curtailment by running the power flow analysis under its current state. Then, the PSDC sends overload relief request and its required load curtailment to the ADMS. The ADMS chooses an appropriate operational scheme for the ADN by running the scheme determination programming, as shown in the right-side part of Fig. 2 . The active reconfiguration scheme is selected as the first layer control. When the active reconfiguration scheme fails to alleviate the overload, the load transferring scheme is selected as the second layer control. Finally, the demand response scheme is conducted as the third layer control, when the above two schemes fail to alleviate the overload in transmission network. After implementing the determined operational scheme, the ADMS calculates the load curtailment from ADN side by running the power flow analysis of the ADN.

Then, the ADMS sends the load curtailment from the ADN side to the PSDC to respond to its overload relief request.

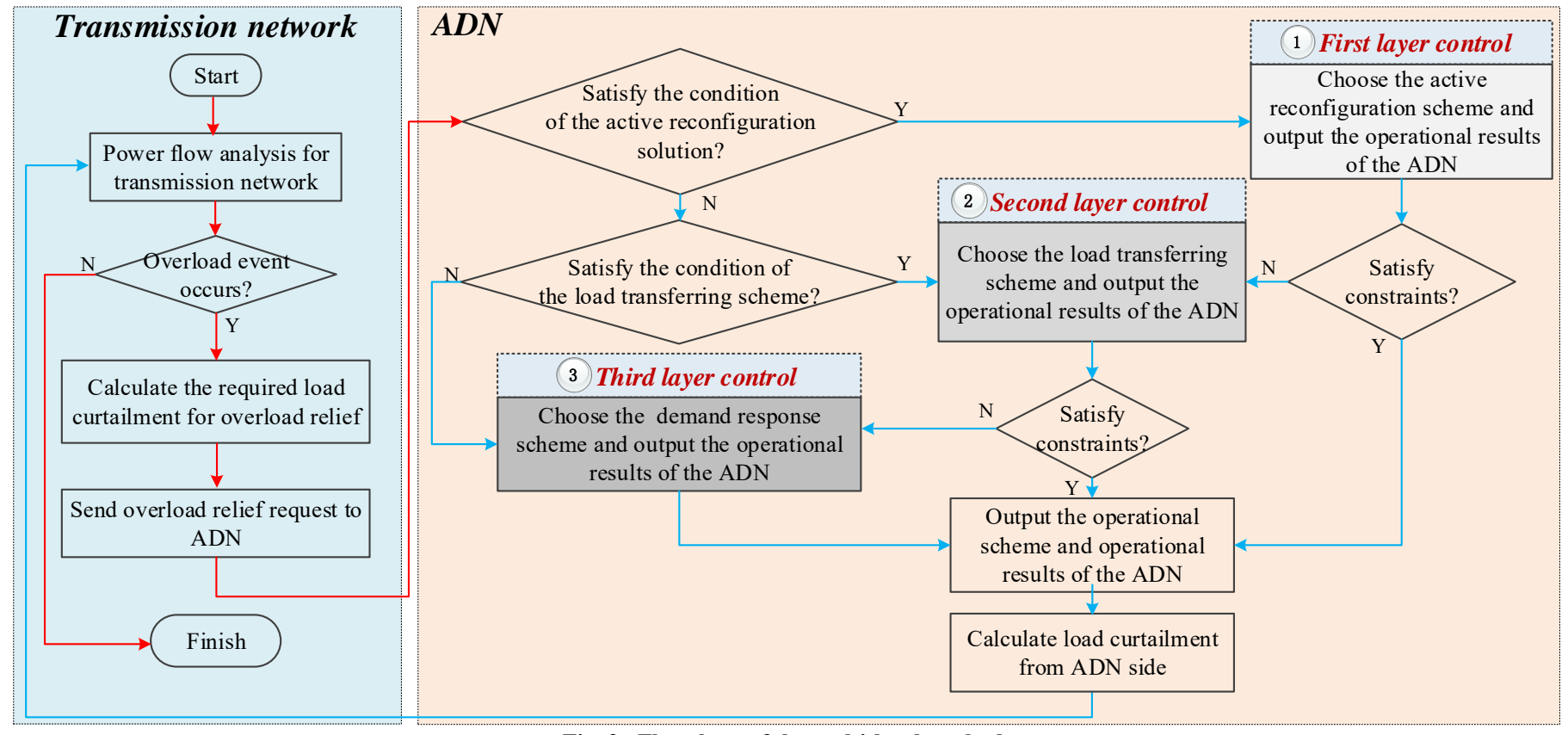

Fig. 2. Flowchart of the multi-level method.

The active reconfiguration scheme aims to reduce the power loss of the ADN by implementing network reconfiguration. Network reconfiguration is implemented to alter the topological structure of the electric feeders of an ADN by changing open/closed status of its sectionalizing and tie switches. Therefore, the optimized network reconfiguration solution of an ADN is determined by searching a binary vector with ' 1 ' assigned to the closed state and ' 0 ' assigned to the open state of each switch using an optimization algorithm. The objective is the minimum power loss of the ADN in this paper. The detailed formulation and solving algorithm of the optimal network reconfiguration of the ADN are presented in Part A of this Section. 
The load transferring scheme aims to transfer proper capacity of loads of the ADN to MGs. The power outputs of MGs are scheduled to accept the transferred loads in a secure manner. After determining the transferred loads from the ADN to a MG, the power output of the MG can be calculated by running the power flow analysis of the MG containing transferred loads [27]. The detailed formulation and solving algorithm of the load transferring scheme are presented in Part B of this Section.

The demand response scheme aims to transfer larger capacity of loads to MGs on the basis of load transferring scheme. In order to accept the increased transferred loads in the MGs, the charging load of EVCS must be curtailed in an optimal way. The power outputs of the MGs are also scheduled to accept the transferred loads from the ADN considering the curtailed charging load of EVCS. In this paper, the objective of the demand response scheme is the minimum shedding capacity of the charging load of EVCS. The detailed formulation and solving algorithm of the load transferring scheme are presented in Part C of this Section.

\section{A. The active reconfiguration scheme}

\section{1) Criteria for scheme selection}

Network reconfiguration is one of the control methods for ADN that changes the operational topology of the ADN by changing the open/close status of its switches. In this paper, the network reconfiguration is used to reduce the power loss of the ADN. The reduced power loss of the ADN is contributed as load curtailment from the ADN side in the active reconfiguration scheme. If the load curtailment contributed by the reduced power loss is above the required load curtailment from the PSDC (as shown in Eq. (1)), the active reconfiguration scheme would be selected.

$$
\Delta P_{e, l} \geq \Delta P_{L D}
$$

Where $\Delta P_{e, l}$ is the reduced power loss of the ADN through network reconfiguration, as shown in Eq. (2). It is contributed as the load curtailment from the $\mathrm{ADN}$ side. $\Delta P_{L D}$ is the required load curtailment from the PSDC, which is calculated by Eq. (3).

$$
\Delta P_{e, l}=P_{\text {loss, before }}-P_{\text {loss, affer }}
$$

Where $P_{\text {loss, before }}$ and $P_{\text {loss, after }}$ are the power loss of the ADN before and after network reconfiguration.

$$
\Delta P_{L D}=\sum_{k=1}^{N_{T}}\left(P_{k}-P_{\lim , k}\right)
$$

Where $P_{k}$ is the active power flow on $k$-th overloaded transmission line, which is calculated by running the power flow analysis of the transmission network under its current state; $P_{\lim , k}$ is the upper limit of the active power flow of the $k$-th overloaded transmission line, which are parameters of the transmission lines of a transmission network. $N_{T}$ is the number of the overloaded transmission lines of the transmission network under its current state. As shown in Eq. (3), the required load curtailment from the PSDC is the sum of overload capacity in each overloaded transmission line. 


\section{2) Scheme solving algorithm}

The switches of the ADN (including sectionalizing and tie switches) have two states: open and closed. Therefore, the status of all the switches can be describe by a binary vector by assigning ' 1 ' to the closed state and ' 0 ' to the open state. The binary particle swarm optimization algorithm (BPSO) is a heuristic algorithm that searches for such binary vectors. Therefore, the BPSO is suitable for solving network reconfiguration problem. It has also been demonstrated to be effective and widely used in [28]-[31] for solving network reconfiguration problem. In this paper, the BPSO is used to solve the feeder reconfiguration problem of the ADN. Other optimization algorithms for solving feeder reconfiguration problem, e.g., hybrid heuristic algorithms [32]-[33], adaptive modified heuristic algorithm [34], mixed-integer convex programming [35], etc., are out of the scope of this paper, which would be studied in our future research work.

To find the optimal solution of the problem, a number of particles are employed. Then, the position of particle $i \boldsymbol{x}_{i}$ and its corresponding flight velocity $\boldsymbol{v}_{i}$ in a $d$-dimensional search space can be denoted in Eq. (4).

$$
\left\{\begin{array}{c}
x_{i d}^{t+1}=x_{i d}^{t}+v_{i d}^{t+1}, i=1,2, \ldots, n, d=1,2, \ldots, m \\
v_{i d}^{t+1}=v_{i d}^{t}+c_{1} * r_{1} *\left(\text { pbset }_{i d}-x_{i d}^{t}\right)+c_{1} * r_{2} *\left(\text { gbset }_{d}-x_{i d}^{t}\right)
\end{array}\right.
$$

Where $n$ is the number of particles in a group; $m$ is the number of members in a particle; $t$ is the iteration number; $c_{1}$ and $c_{2}$ are acceleration constants; $r_{1}$ and $r_{2}$ are uniformly distributed random numbers in $[0,1] ; v_{i d}^{t}$ is velocity of particle $i$ at iteration $t, V_{d}^{\min } \leq$ $v_{i d}^{t} \leq V_{d}^{\max } ; x_{i d}^{t}$ is current position of particle $i$ at iteration $t$; pbest $t_{i d}$ is the best position of particle $i$; gbest $t_{d}$ is the global best position. As shown in Eq. (4), the movement of each particle toward the optimal solution is guided by the knowledge from itself and other particles. The elements of the position vector $\boldsymbol{x}_{i}$ at iteration $t$ are determined according to Eq. (5):

$$
x_{i d}^{t}=\left\{\begin{array}{cc}
1, & \text { if } \rho_{i d}<s\left(v_{i d}\right) \\
0, & \text { otherwise }
\end{array}\right.
$$

where $\rho_{i d}$ is a random number in the range of $[0,1] ; s\left(v_{i d}\right)$ is a sigmoidal function defined in Eq. (6):

$$
s\left(v_{i d}\right)=\frac{1}{1+\exp \left(-v_{i d}\right)}
$$

In this paper, The BPSO algorithm searches the optimal status of switches of the ADN to minimize its power loss. Therefore, the objective of the BPSO is the minimum power loss of the ADN, as shown in Eq. (7).

$$
\min f=\min P_{\text {loss }, \text { affer }}=\min \left\{\sum_{i=1}^{N_{\text {Branch }}} R_{i} \times\left|I_{i}\right|^{2}\right\}
$$

Where $R_{i}, I_{i}$ are the resistance and current of the $i$-th branch of the $\mathrm{ADN} ; N_{\mathrm{Branch}}$ is the number of branches of the ADN after network reconfiguration.

The constraints include the power flow equation of the ADN, limits of bus voltage amplitude, limits of branch current and the 
constraint of radial topology of the ADN, as shown in Eqs. (8) (12):

$$
\begin{gathered}
P_{i}=e_{i} \sum_{j \in n_{k}, j \neq i}\left(G_{i j} e_{j}-B_{i j} f_{j}\right)+f_{i} \sum_{j \in n_{k}, j \neq i}\left(G_{i j} f_{j}+B_{i j} e_{j}\right) \quad i \in n_{k} \\
Q_{i}=f_{i} \sum_{j \in n_{k}, j \neq i}\left(G_{i j} e_{j}-B_{i j} f_{j}\right)-e_{i} \sum_{j \in n_{k}, j \neq i}\left(G_{i j} f_{j}+B_{i j} e_{j}\right) \quad i \in n_{k} \\
V_{i, \min }^{2}<\left(e_{i}^{2}\right)+\left(f_{i}^{2}\right)<V_{i, \text { max }}^{2} \quad i \in n_{k} \\
\left(I_{i j}\right)^{2}=\left(G_{i j}{ }^{2}+B_{i j}{ }^{2}\right) \times\left[\left(V_{i}^{2}+V_{j}^{2}\right)-2\left(e_{i} e_{j}+f_{i} f_{j}\right)\right] \leq\left(I_{i j, \max }\right)^{2} \quad i j \in n_{l} \\
n_{\text {loop }}=n_{l}-n_{k}+1
\end{gathered}
$$

where $n_{k}$ is the total number of buses of the $\mathrm{ADN} ; P_{i}$ is the active power injection at bus $v_{i}$; $Q_{i}$ is the reactive power injection at bus $v_{i}$; $V_{i}$ is voltage amplitude at bus $v_{i} ; e_{i}$ and $f_{i}$ are the real and imaginary parts of the complex voltage at bus $v_{i} ; Y_{i j}=G_{i j}+j B_{i j}$ is the $i j$-th element of the node admittance matrix of the $\mathrm{ADN} ; V_{i, \max }$ and $V_{i, \text { min }}$ are the upper and lower limits of voltage amplitude limits; $I_{i j}$ is the current of branch $i j ; I_{i j \text {, max }}$ is upper current limit of branch $i j ; n_{l}$ is the total number of branches of the ADN; $n_{\text {loop }}$ is the number of loops of the ADN topology.

The flowchart of the active reconfiguration problem based on BPSO algorithm is shown in Fig. 3. The algorithm is stopped if one of the following stopping criteria is satisfied:

(1) The number of iterations exceeds its limit.

(2) The optimal value keeps unchanged within 10 iterations.

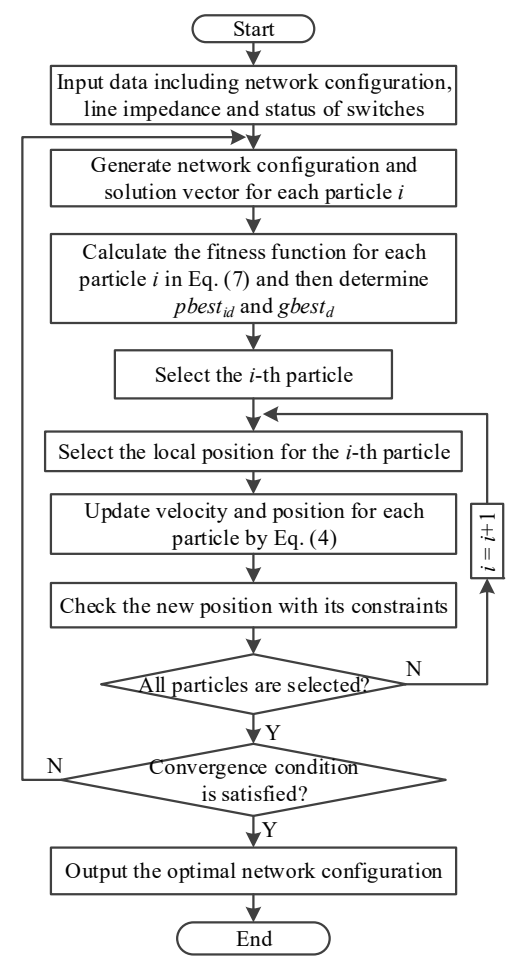

Fig. 3. flowchart of the active reconfiguration problem based on BPSO algorithm. 


\section{B. The load transferring scheme}

\section{1) Criteria for scheme selection}

When Eq. (13) is met, the load transferring scheme would be selected:

$$
\Delta P_{e, l} \leq \Delta P_{L D}
$$

where $\Delta P_{e, l}$ is the same parameter as in Eq. (1), which is the reduced power loss of the ADN through network reconfiguration. Therefore, Eq. (13) is used to decide whether the active reconfiguration scheme can alleviate the overload of the transmission network.

\section{2) Scheme solving algorithm}

A rooted tree method is used to develop the hierarchical model of the topology of the distribution network according to its radial structure. Then, the depth first search (DFS) algorithm [6], [12] is used to decide the initial load transferring scheme of the node-weight rooted tree. The final load transferring scheme is finally determined by checking several feasible constraints step by step [12]. The steps for determining the load transferring scheme are given as follows:

Step 1) Develop the hierarchical model of the topology of the distribution network.

An electric feeder is modelled as a five-layer hierarchical rooted tree $\boldsymbol{T}(\boldsymbol{V}, \boldsymbol{E})$, as shown in Fig. 4. The electric buses and electric branches of the electric feeder are modelled as tree nodes and tree branches of a rooted tree $\boldsymbol{T}(\boldsymbol{V}, \boldsymbol{E})$, where $\boldsymbol{V}=\left\{v_{1}, \ldots, v_{\mathrm{n}}\right\}$ is the set of tree nodes, in which $n$ represents the total number of nodes of the tree; $\boldsymbol{E}=\left\{e_{12}, \ldots, e_{i j}, \ldots, e_{37}\right\}$ is the set of tree branches, in which $e_{i, j}(i<j)$ is the branch linking the node $v_{i}$ and the node $v_{j}$.

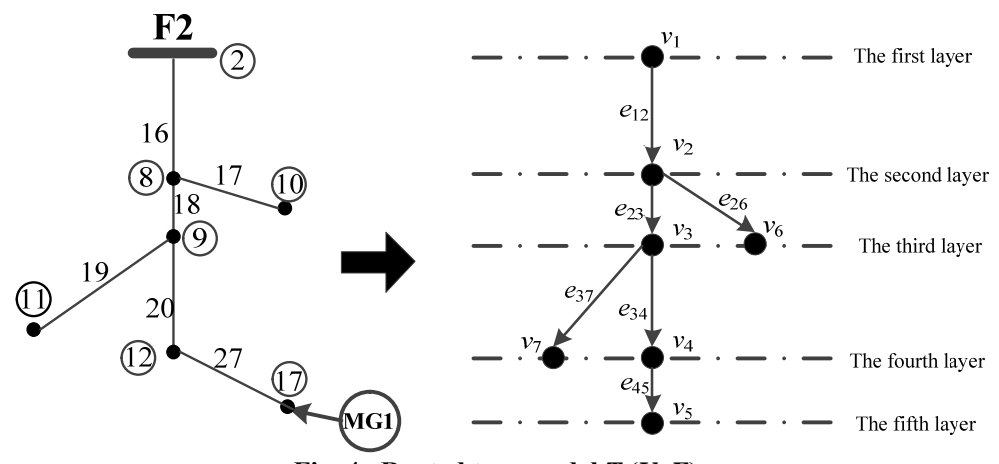

Fig. 4. Rooted tree model $T(V, E)$.

Step 2) Define the node weights of $\boldsymbol{T}(\boldsymbol{V}, \boldsymbol{E})$.

The weights of nodes in $\boldsymbol{T}(\boldsymbol{V}, \boldsymbol{E})$ are defined as the active power injection to the electric buses, as depicted in Eq. (14). $\mathbf{W}=\left\{w_{1}, \ldots w_{i}, \ldots, w_{\mathrm{n}}\right\}$ is the vector of the node weights.

$$
w\left(v_{i}\right)_{i \in \Omega_{j}}=\bar{P}_{\mathrm{MG}_{j}}-P_{L i}
$$


Where $\bar{P}_{\mathrm{MG}_{i}}$ is the maximum power output of MG $j$ connecting to node $v_{i} ; P_{L i}$ is active load of node $v_{i}$; $w\left(v_{i}\right)$ is the node weight of node $v_{i}$

Step 3) Search the initial load transferring scheme.

DFS algorithm is employed to search the node-weighted rooted subtree $\boldsymbol{T}_{s}\left(\boldsymbol{V}_{s}, \boldsymbol{E}_{s}\right)$, i.e. the initial load transferring scheme, in the node-weight rooted tree $\boldsymbol{T}(\boldsymbol{V}, \boldsymbol{E})$. Algorithm 1 illustrates how the DFS-based algorithm is implemented to search the initial load transferring scheme.

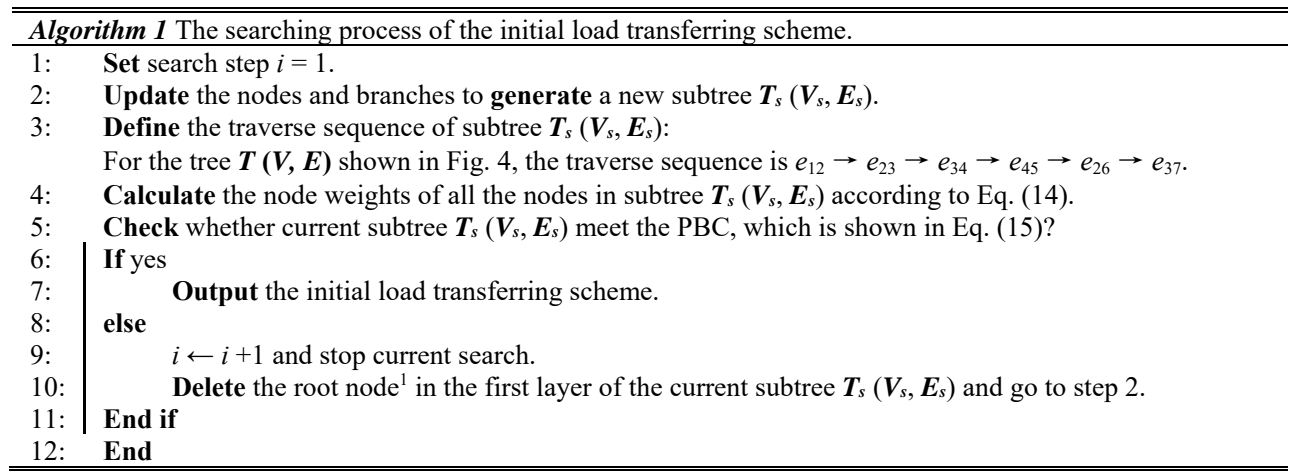

The Power balance constraint (PBC) of the subtree $\boldsymbol{T}_{s}\left(\boldsymbol{V}_{s}, \boldsymbol{E}_{s}\right)$ is the criteria for stopping the search process of the initial load transferring scheme, as shown in line 5 of Algorithm 1. PBC is the sum of node weights of all the buses in the subtree $\boldsymbol{T}_{s}\left(\boldsymbol{V}_{s}, \boldsymbol{E}_{s}\right)$, as shown in Eq. (15).

$$
F\left(T_{S}\right)=\sum_{i \in \Omega_{j}} w\left(v_{i}\right)=\sum_{i \in \Omega_{j}}\left(\bar{P}_{\mathrm{MGj}}-P_{L i}\right)>0
$$

where $F\left(T_{S}\right)$ is the evaluation function of the PBC.

The flowchart of the search process for the initial load transferring scheme is shown in Part 1 in Fig. 5. The initial load transferring scheme only considers the PBC constraint. Other constraints, i.e., Eqs. (16) (19), are considered in the feasibility check stage to determine the final load transferring scheme.

Step 4) Decide the final load transferring scheme.

Check the constraints from Eq. (16) to Eq. (19), and if all the constraints are met, the final load transferring scheme is decided. However, if one of the constraints is not met, delete the root node of the rooted subtree $\boldsymbol{T}_{s}\left(\boldsymbol{V}_{s}, \boldsymbol{E}_{s}\right)$ of the initial load transferring scheme, which is obtained in Step 3). Then, a new rooted tree $\boldsymbol{T}(\boldsymbol{V}, \boldsymbol{E})$ is generated. Then, turn to step 3) to search the load transferring scheme again until the final scheme is determined. The flowchart of the load transferring scheme including the initial search and feasibility check is shown in Fig. 5.

\footnotetext{
${ }^{1} \mathrm{~A}$ root node of a tree is the first node in the top layer of the tree. The root node of the rooted tree $T(V, E)$ in Fig. 4 is $v_{1}$.
} 


\section{Constraints of the feasibility check for the final load transferring scheme:}

- Constraint of rated value and limit (RLC):

$$
\left|P_{e i j}\right|_{e i j \in V}<\alpha * P_{\text {rated_eij }}
$$

where $P_{e i j}$ is the active power flow of the branch $e_{i j} ; P_{\text {rate } \_i j}$ is the maximum capacity of the branch $e_{i j}$; $\alpha$ is the margin coefficient.

- Constraint of radial structure:

$$
N=M+1
$$

where $N$ is the number of the buses of the MG containing transferred loads; $M$ is the number of branches.

- Load transferring range constraint:

The layer number of buses transferred to the MG should be higher than that outside of the MG, as shown in Eq. (18). In this case, outage loads in the distribution network after implementing the load transferring scheme are avoided.

$$
\operatorname{depth}\left(v_{i}, i \in \mathbf{\Omega}\right) \geq \operatorname{depth}\left(v_{j}, j \notin \mathbf{\Omega}\right)
$$

Where $\operatorname{depth}\left(v_{i}, i \in \mathbf{\Omega}\right)$ is the layer number of bus $v_{\mathrm{i}}$ transferred to the MG.

- Demand constraint:

$$
\Delta P_{L D} \leq \sum_{i \in \Omega_{j}} P_{L i} \leq k_{D} \Delta P_{L D}
$$

where $k_{D}$ is the margin coefficient. The constraint is developed to avoid load over-shedding of the ADN in the load transferring scheme. 


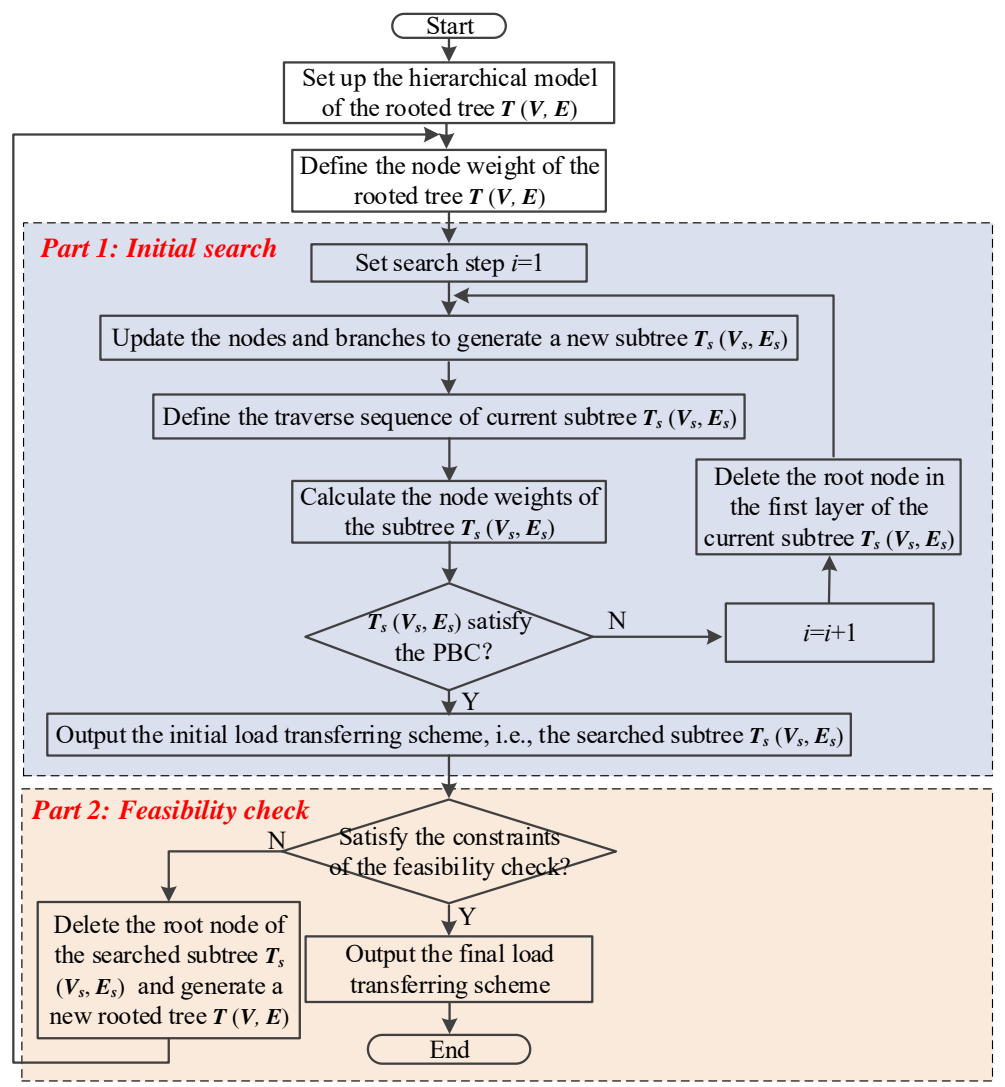

Fig. 5. Flowchart of the load transferring scheme.

\section{The demand response scheme}

\section{1) Criteria for scheme selection}

When Eq. (20) is met, the demand response scheme would be selected:

$$
\sum_{j=1}^{m}\left(\sum_{i \in \Omega_{j}} P_{L i}\right) \leq \Delta P_{L D}
$$

where $\sum_{i \in \Omega_{j}} P_{L i}$ is the total capacity of transferred loads from the ADN to the MG obtained in the load transferring scheme, as

\section{illustrated in Part B of Section III.}

\section{2) Solving algorithm}

The demand response scheme utilizes the reducible EV charging loads to transfer larger capacity of loads to the MGs on the basis of load transferring scheme. It further increases the load curtailment from the ADN side to alleviate the overload in transmission network. The problem is decomposed into two sub problems in demand response scheme, as shown in Fig. 6. Problem I aims to determine the capacity of transferred loads from the ADN to the MGs, which is contributed as load curtailment from the ADN side in the demand response scheme. Problem II aims to determine the capacity of EVs load shedding from the EVCS to 
guarantee the secure and reliable operation of the MG containing the transferred loads from the ADN.

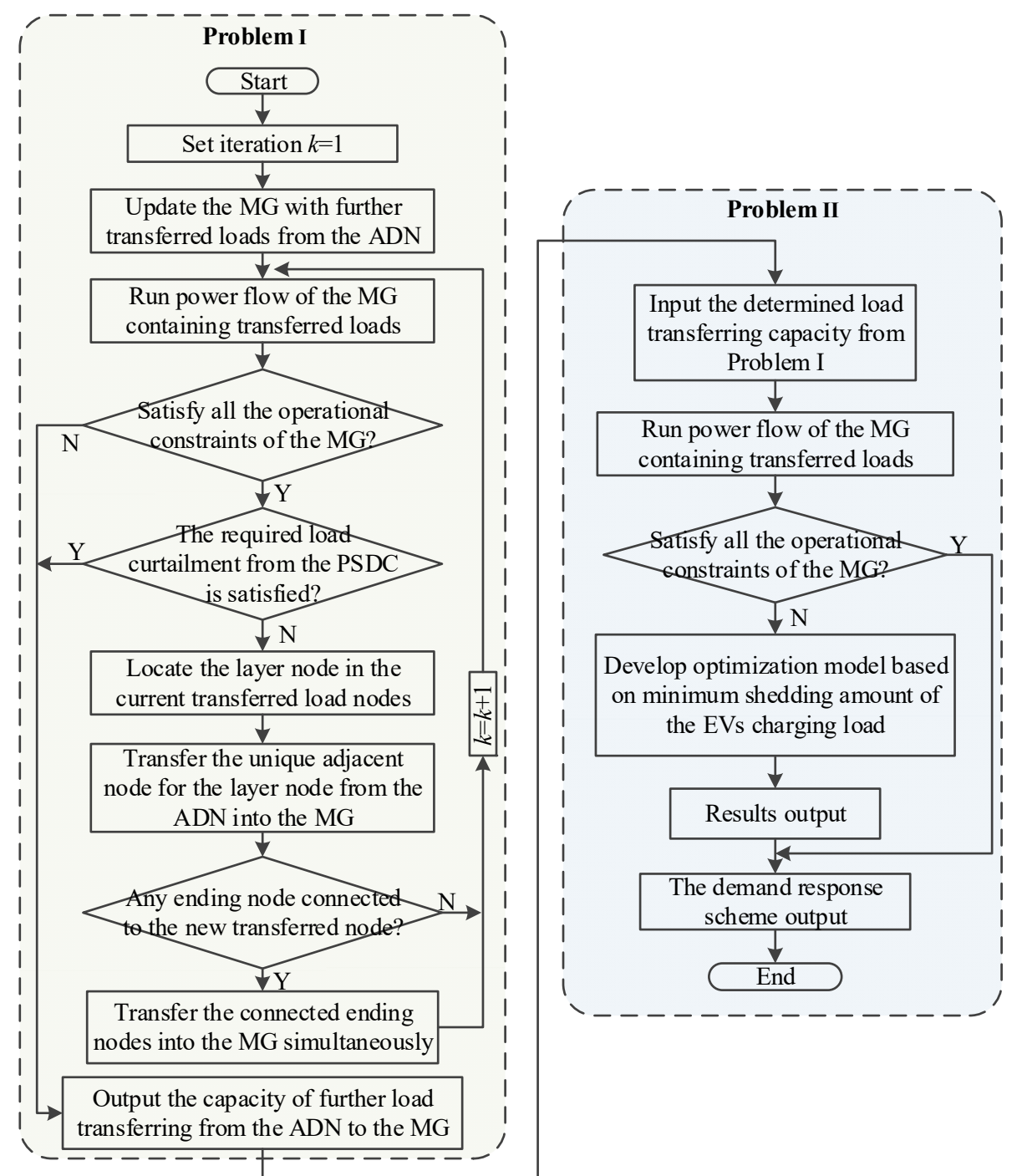

Fig. 6. Flow chart of the demand response scheme.

- Problem I:

The first sub-problem is developed to decide the capacity of further load transferring from the ADN to the MGs on the basis of load transferring scheme. The capacity of further load transferring from the ADN to each MG can be determined by implementing Algorithm 2. The layer node and the adjacent node [36] mentioned in Algorithm 2 are indicated in Fig. 7.

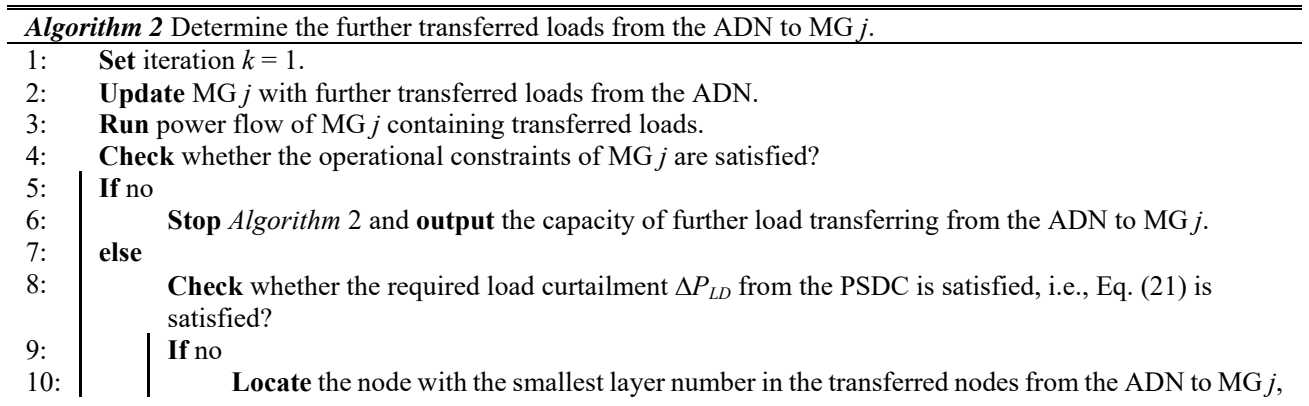




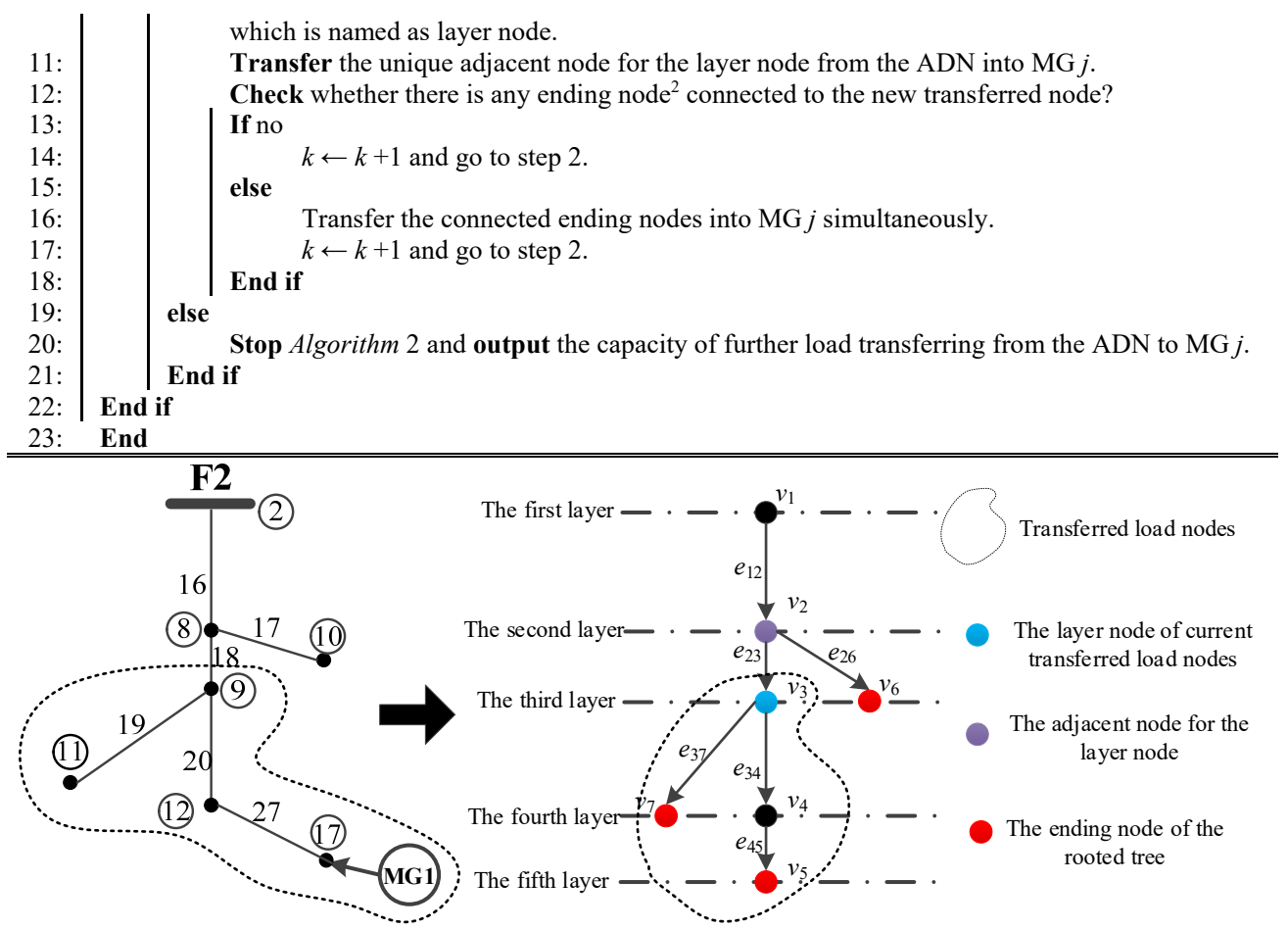

Fig. 7. Schematic of the layer node, the adjacent node and the ending node in the rooted tree $T(V, E)$.

$$
\sum_{j=1}^{m}\left(\sum_{i \in \Omega_{j}} P_{L i}+\sum_{\substack{k=1 \\ k \in \Omega_{j}}}^{N_{L}} P_{k}\right)_{j} \geq \Delta P_{L D}
$$

Where $P_{k}$ is the active load of the further transferred node from the ADN to $\mathrm{MG} j ; N_{L}$ is the number of the further transferred nodes from the $\mathrm{ADN}$ to $\mathrm{MG} j ; m$ is the number of MGs. For the details of the power flow model of a radial electric network, one can referred to [37] due to limited space. It is worth noting that the power output of MG $j$ and the shedding capacity of EV charging load from each EVCS in the scope of $\mathrm{MG} j$ are considered as their upper limits in the power flow analysis for $\mathrm{MG} j$ containing transferred loads. In this case, $\mathrm{MG} j$ can accept transferred loads from the ADN as much as possible.

- Problem II:

The second sub-problem is developed to decide the capacity of EVs load shedding from the EVCS to guarantee the secure and reliable operation of each MG. For each $\mathrm{MG} j$ containing transferred loads, the objective function is the minimum shedding amount of the EVs charging load in MG $j$, which is shown in Eq. (22):

$$
\min \sum_{\substack{p=1 \\ p \in \Omega_{j}}}^{N_{p}} P_{E V, p}
$$

where $N_{p}$ is the number of EVCS in the scope of MG $j ; P_{E V, p}$ is the shedding capacity of EV charging load from EVCS $p$.

\footnotetext{
${ }^{2}$ An ending node of a tree is the terminated node on a branch, where no descendent node can be found on the same branch following the defined traverse sequence [6]. The ending nodes of the rooted tree $T(V, E)$ in Fig. 4 are $v_{5}, v_{6}$ and $v_{7}$.
} 
The constraints include the limit of active power output of MG $j$ (Eq. (23)), the limit of reactive power output of MG $j$ (Eq. (24)), limit of shedding capacity of EV charging load from EVCS $p$ (Eq. (25)).

$$
\begin{array}{cl}
P_{M G_{, \text {,inn }}} \leq P_{M G_{j}} \leq P_{M G_{j, \text { max }}} & j=1,2, \ldots, m \\
Q_{M G_{, \text {,ini }}} \leq Q_{M G_{j}} \leq Q_{M G_{j, \text { max }}} & j=1,2, \ldots, m \\
P_{E V, p} \leq \bar{P}_{E V, p} & p \in \Omega_{j}
\end{array}
$$

Where $P_{M G j}$ and $Q_{M G j}$ are active and reactive power output from MG $j ; P_{M G j \max }, P_{M G j, \min }, Q_{M G j \text { max }}$ and $Q_{M G j \text { min }}$ are the corresponding upper and lower limits of power output from $\mathrm{MG} j ; \bar{P}_{E V, p}$ is the upper limit of reducible EV charging load of the $\operatorname{EVCS} p$.

The constraints also include the power flow equations, limits of bus voltage amplitude, limits of branch current and constraint of radial topology of MG $j$ containing the transferred loads and the constraint of its radial structure, which are described similar to Eqs. (8) (12). The specific equations are not presented here due to limited space.

\section{CASE STUdies}

The IEEE 30-bus system [38] with an ADN connecting to bus 19 is carried out to verify the proposed multi-level method in this paper, as shown in Fig. 8. The thermal limits of the transmission lines of the IEEE 30-bus system can be found in [39]. It is assumed that a fault occurs at transmission line $L_{18-19}$ leading to overload in line $L_{19-20}$. Two distribution networks are tested as the ADN respectively.

\section{A. Case I}

A three-feeder distribution network [40] with one MG and two EVCSs is shown in Fig. 8 (b). The MG locates at bus 17 and two EVCSs locate at bus 8,12 respectively. The maximum power output of the MG is set to be $13+\mathrm{j} 10 \mathrm{MVA}$. The maximum EV charging load of EVCS1 and EVCS2 are set to be $4 \mathrm{MW}$ and 4.5MW respectively. The margin coefficient $k_{D}$ and $\alpha$ are set to be 1.2 and 0.95 respectively. TABLE I shows the chosen values of the parameters for the BPSO [28].

TABLE I

PARAMETERS OF BPSO

\begin{tabular}{cc}
\hline \hline Parameters & Value \\
\hline Number of particles & $10 \times$ No. of switches \\
$c_{1}$ & 2 \\
$c_{2}$ & 2 \\
Maximum number of iterations & $10 \times$ No. of switches \\
\hline
\end{tabular}




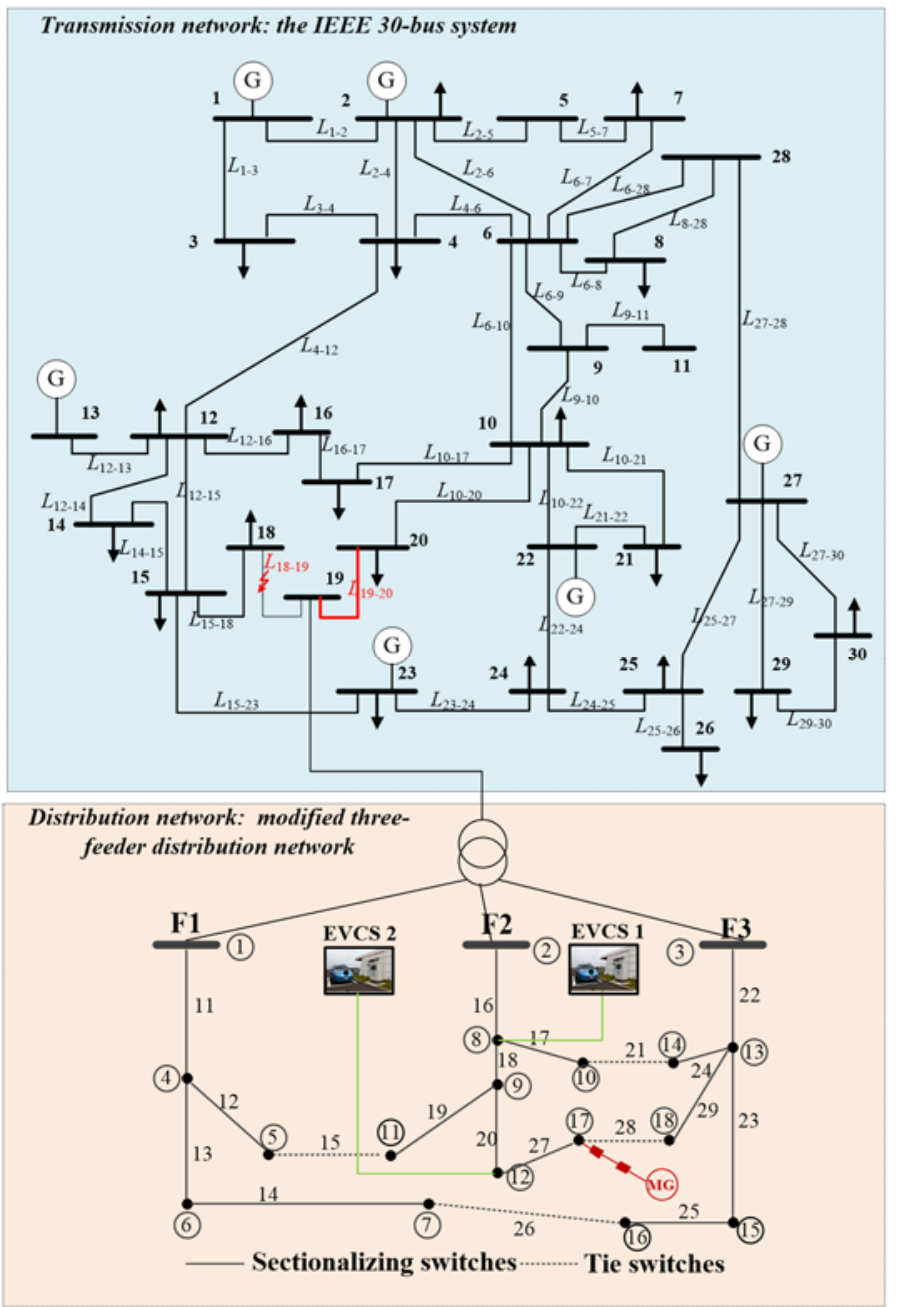

Fig. 8. Test system of Case I.

\section{1) Scenario 1: Simulation results for the active reconfiguration scheme}

When a fault occurs at $L_{18-19}$, the power flow of $L_{19-20}$ is $32.02 \mathrm{MW}$ (obtained by running power flow of the transmission network), which violates its thermal limit (32MW). The $\Delta P_{L D}$ is $0.02 \mathrm{MW}$ and the $\Delta P_{e, l}$ is $0.0242 \mathrm{MW}$ in this scenario (obtained through power flow calculation, as shown in TABLE II), which meets the Eq. (1). Therefore, the active reconfiguration scheme is selected.

The results of feeder reconfiguration of the ADN are shown in TABLE II. The power flow of $L_{19-20}$ is $31.98 \mathrm{MW}$ after conducting the active reconfiguration scheme, which is below its thermal limit and the overload on $L_{19-20}$ is alleviated.

TABLE II

FEEDER RECONFIGURATION RESULTS OF THE ADN IN SCENARIO 1

\begin{tabular}{cccc}
\hline \hline & Open Switches & Line Loss (MW) & Loss reduction (\%) \\
\hline Before Reconfiguration & $15,21,26,28$ & 0.7260 & - \\
After Reconfiguration & $19,21,26,28$ & 0.7018 & 3.33 \\
\hline \hline
\end{tabular}




\section{2) Scenario 2: Simulation results for the load transferring scheme}

The thermal limit of line $L_{19-20}$ is set to be $85 \%$ of its original value in Scenario 2, which is 27.2MW. If the ADMS choose the active reconfiguration scheme to alleviate the overload in transmission network in this scenario, the power flow of line $L_{19-20}$ would be $31.98 \mathrm{MW}$. In this case, it violates the thermal limit of line $L_{19-20}$. Therefore, the active reconfiguration scheme fails to solve the overload problem and the load transferring scheme is chosen. DFS of node-weight rooted tree is conducted to search the initial load transferring scheme firstly, and the final load transferring scheme is then decided after the feasibility check.

Fig. 9 illustrates the DFS algorithm for initial load transferring scheme in the ADN. Firstly, the electric feeder F2 of the ADN is modelled as a five-layer hierarchical rooted tree ${ }^{3}$, as shown in the left side figure in Fig. 9. Then, the initial load transferring scheme can be obtained by implementing the following search steps:

1): Generate current rooted tree $\boldsymbol{T}(\boldsymbol{V}, \boldsymbol{E})$, where $\boldsymbol{V}=\left\{v_{1}, v_{2}, v_{3}, v_{4}, v_{5}, v_{6}, v_{7}\right\}$ and $\boldsymbol{E}=\left\{e_{12}, e_{23}, e_{34}, e_{45}, e_{26}, e_{37}\right\}$.

2): Define the traverse sequence of current rooted tree $\boldsymbol{T}(\boldsymbol{V}, \boldsymbol{E})$, which is $e_{12} \rightarrow e_{23} \rightarrow e_{34} \rightarrow e_{45} \rightarrow e_{26} \rightarrow e_{37}$.

3): Calculate the node weights of all the nodes in current rooted tree, i.e., $\boldsymbol{W}=\left\{w_{1}, w_{2}, w_{3}, w_{4}, w_{5}, w_{6}, w_{7}\right\}$. The evaluation function of the PBC of current rooted tree is the sum of all the node weights, which is $-2.1 \mathrm{MW}$.

4): Check whether the current rooted tree meet the PBC in Eq. (15). Apparently, it fails to meet the PBC $(-2.1 \mathrm{MW}<0)$ and the search process is conducted continuously along the defined traverse sequence.

5): Delete the root node of current tree (i.e., $\left.v_{1}\right)$ and generate a new rooted subtree $\boldsymbol{T}_{s}\left(\boldsymbol{V}_{\boldsymbol{s}}, \boldsymbol{E}_{s}\right)$, where $\boldsymbol{V}_{\boldsymbol{s}}=\left\{v_{2}, v_{3}, v_{4}, v_{5}, v_{6}, v_{7}\right\}$ and $\boldsymbol{E}_{s}=\left\{e_{23}, e_{34}, e_{45}, e_{26}, e_{37}\right\}$. The root node of the new rooted subtree is $v_{2}$.

6): Define the traverse sequence of current rooted subtree $\boldsymbol{T}_{\boldsymbol{s}}\left(\boldsymbol{V}_{\boldsymbol{s}}, \boldsymbol{E}_{\boldsymbol{s}}\right)$, which is $e_{23} \rightarrow e_{34} \rightarrow e_{45} \rightarrow e_{26} \rightarrow e_{37}$.

7): Calculate the node weights of all the nodes in current rooted subtree, i.e., $W_{s}=\left\{w_{2}, w_{3}, w_{4}, w_{5}, w_{6}, w_{7}\right\}$. The evaluation function of the PBC of current rooted tree is the sum of all the node weights, which is $-2.1 \mathrm{MW}$.

8): Check whether the current rooted subtree meet the PBC in Eq. (15). Apparently, it fails to meet the PBC $(-2.1 \mathrm{MW}<0)$ and the search process is conducted continuously along the defined traverse sequence.

9): Delete the root node of current subtree (i.e., $\left.v_{2}\right)$ and generate a new rooted subtree $\boldsymbol{T}_{\boldsymbol{S}}^{*}\left(\boldsymbol{V}_{\boldsymbol{S}}^{*}, \boldsymbol{E}_{\boldsymbol{S}}^{*}\right)$, where $\boldsymbol{V}_{\boldsymbol{S}}^{*}=\left\{v_{3}, v_{4}, v_{5}, v_{7}\right\}$ and $\boldsymbol{E}_{\boldsymbol{S}}^{*}=\left\{e_{34}, e_{45}, e_{37}\right\}$. The root node of the new rooted subtree is $v_{3}$.

10): Define the traverse sequence of current rooted subtree $\boldsymbol{T}_{S}^{*}\left(\boldsymbol{V}_{\boldsymbol{S}}^{*}, \boldsymbol{E}_{S}^{*}\right)$, which is $e_{34} \rightarrow e_{45} \rightarrow e_{37}$.

${ }^{3}$ There is only one MG connected in the ADN, which is located on feeder F2. Therefore, only feeder F2 is modelled as a rooted tree to search the initial load transferring scheme. 
11): Calculate the node weights of all the nodes in current rooted subtree, i.e., $\boldsymbol{W}_{s}^{*}=\left\{w_{3}, w_{4}, w_{5}, w_{7}\right\}$. The evaluation function of the $\mathrm{PBC}$ of current rooted tree is the sum of all the node weights, which is $2.9 \mathrm{MW}$.

12): Check whether the current rooted subtree meet the PBC in Eq. (15). Apparently, it meets the PBC (2.9 MW $>0)$ and the search process is stopped.

The initial load transferring scheme is then obtained, as shown in the circled area in Fig. 9. As highlighted in red color in Fig. 9, the initial load transferring scheme is isolated between node $v_{2}$ and $v_{3}$, i.e., between bus 8 and 9 of feeder F2. The change of the rooted trees during the search process of the initial load transferring scheme in electric feeder F2 is shown in Fig. 10. It can be observed that the rooted tree is updated continuously until a subtree meeting PBC is obtained during the search process.

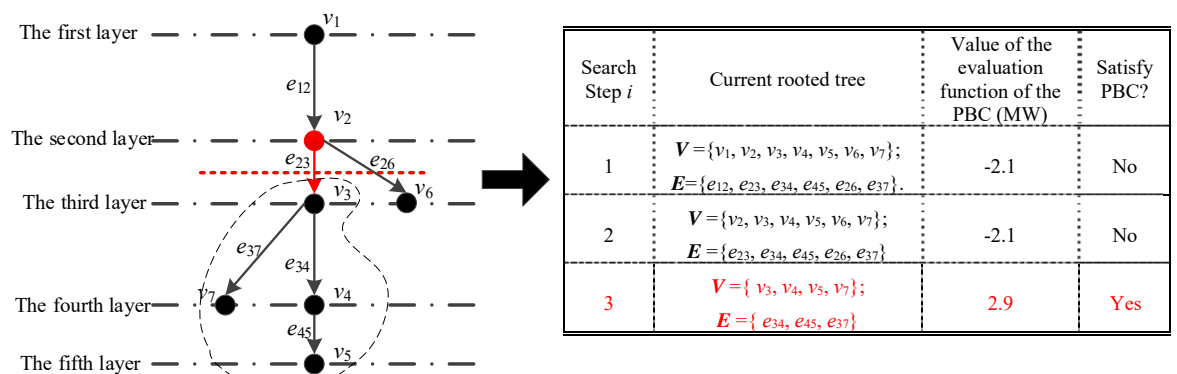

Fig. 9. DFS algorithm for initial load transferring scheme in feeder F2 of the ADN in Case I.

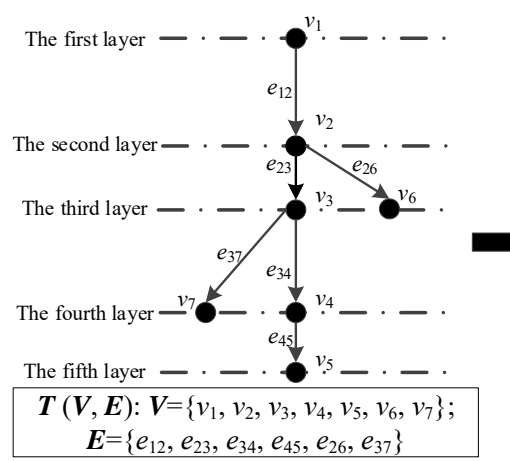

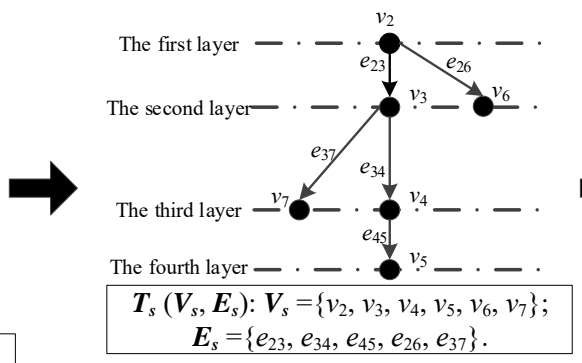

(b)

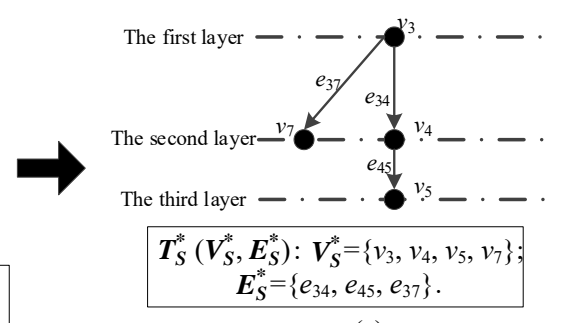

(c)

(a)

Fig. 10. Change of the rooted trees during the search process of the initial load transferring scheme in electric feeder F2: (a) the initial rooted tree, (b) subtree after deleting root node $v_{1}$, (c) subtree after deleting root node $v_{2}$, i.e., the initial load transferring scheme

Then, the feasibility of the obtained initial load transferring is checked with the constraints from Eqs. (16) (19). After transferring the initial capacity of loads to the MG, the number of buses of the ADN changed from 16 to 12 ; the number of sectionalizing switches changed from 15 to 11 ; the number of tie switches changed from 4 to 2 . The power flow of line $L_{19-20}$ is 20.18MW, while the $\Delta P_{L D}$ is $4.82 \mathrm{MW}$ and the $\sum P_{L i}$ is $11.1 \mathrm{MW}^{4}$. Therefore, the initial load transferring scheme fails to meet the constraint Eq. (19) with the $k_{D}$ of 1.2. As a result, the root node of the rooted subtree of the initial load transferring scheme, i.e., the subtree shown in Fig. 10 (c), is deleted to generate a new rooted subtree. Then, search the initial load transferring scheme again in 
the new generated rooted subtree until a final load transferring scheme meeting all the constraints is obtained. The schemes including the initial load transferring scheme and the final load transferring scheme are shown in Fig. 11. It can be observed that the range of transferred loads is narrowed from the initial scheme to the final scheme.

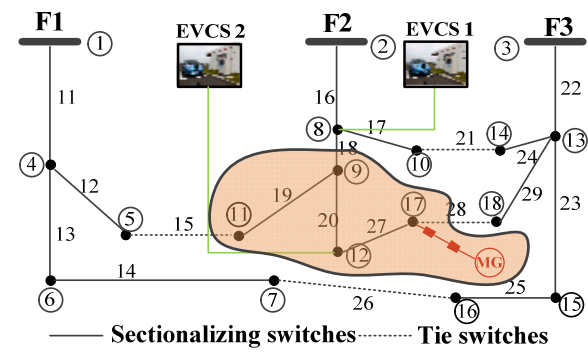

(a) The initial load transferring scheme

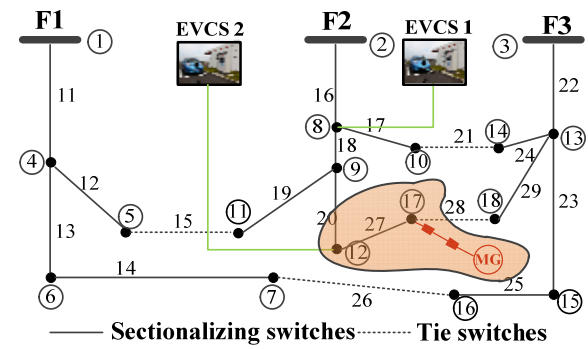

(b) The final load transferring scheme

Fig. 11. The load transferring scheme in Case I: (a) initial; (b) final.

After conducting the final load transferring scheme (as shown in Fig. 11 (b)), the power flow of line $L_{19-20}$ is reduced to 26.06MW, which is below its thermal limit. The $\Delta P_{L D}$ is $4.82 \mathrm{MW}$ and the updated $\sum P_{L i}$ is $5.5 \mathrm{MW}$, which meets the constraint (19), i.e. there is no load over-shedding from the ADN. The capacity of the final transferred loads is lower than the its initial value (shown in Fig. 11 (a) and (b)), which can explain this issue too. Other constraints are also met after the feasibility checking. Therefore, the final load transferring scheme is determined and the operational results of the MG containing transferred loads are shown in TABLE III.

TABLE III

RESULTS OF THE LOAD TRANSFERRING SCHEME IN CASE I

\begin{tabular}{|c|c|c|c|}
\hline Power Output from MGs (MVA) & Load Shedding from EVCS1 (MW) & Load Shedding from EVCS2 (MW) & Line Loss (MW) \\
\hline $5.511+\mathrm{j} 1.189$ & 0 & 0 & 0.011 \\
\hline
\end{tabular}

\section{3) Scenario 3: Simulation results for the demand response scheme}

The thermal limit of line $L_{19-20}$ is set to be $60 \%$ of its original value in Scenario 3, which is $19.2 \mathrm{MW}$. If the ADMS choose the load transferring scheme to alleviate the overload in transmission network in this scenario, the power flow of line $L_{19-20}$ would be 26.06MW (obtained in Scenario 2). In this case, it violates the thermal limit of line $L_{19-20}$. Therefore, the load transferring scheme fails to solve the overload problem and the demand response scheme is chosen. 
Problem I is solved firstly to obtain the capacity of further load transferring from the ADN to the MG on the basis of load transferring scheme. It can be solved by implementing Algorithm 2 as follows:

1): Set iteration $k=1$.

2): Update the MG with the transferred loads from the ADN. The updated MG is shown in the circled area in Fig. 11 (b) and Fig. 12 (a).

3): The operational constraints of the updated MG are satisfied by running power flow analysis.

4): Check whether the required load curtailment $\Delta P_{L D}$ from the PSDC is satisfied in iteration $k=1$. In scenario 3, the required load curtailment from the PSDC is $\Delta P_{L D}=P_{k}-P_{\text {lim, }, k}=32.02 \mathrm{MW}-19.2 \mathrm{MW}=12.82 \mathrm{MW}$. The capacity of current transferred loads is $5.5 \mathrm{MW}$ in current iteration, which is contributed as load curtailment from the ADN side. Apparently, the required load curtailment from the PSDC is not satisfied, i.e., Eq. (21) is not satisfied.

5): Locate the layer node in the updated MG, which is node $v_{4}$, i.e., bus 12 in feeder F2, as highlighted in blue color in Fig. 12 (a).

6): Transfer the unique adjacent node, i.e., node $v_{3}$ (bus 9), for the layer node $v_{4}$ (bus 12) from the ADN into the MG.

7): There is an ending node $v_{7}$ (bus 11) connected to the new transferred node $v_{3}$. Therefore, the ending node $v_{7}$ is also transferred from the ADN to the MG.

8): Set iteration $k=k+1=2$.

9): Update the MG with the further transferred loads from the ADN. The updated MG is shown in the circled area in Fig. 12 (b).

10): The operational constraints of the updated MG are satisfied by running power flow analysis.

11): Check whether the required load curtailment $\Delta P_{L D}$ from the PSDC is satisfied in iteration $k=2$. In scenario 3, the required load curtailment from the PSDC is $\Delta P_{L D}=P_{k}-P_{\lim , k}=32.02 \mathrm{MW}-19.2 \mathrm{MW}=12.82 \mathrm{MW}$. The capacity of current transferred loads is 11.1 MW in current iteration, which is contributed as load curtailment from the ADN side. Apparently, the required load curtailment from the PSDC is not satisfied, i.e., Eq. (21) is not satisfied.

12): Locate the layer node in the updated MG, which is node $v_{3}$, i.e., bus 9 in feeder F2, as highlighted in blue color in Fig. 12 (b).

13): Transfer the unique adjacent node, i.e., node $v_{2}$ (bus 8), for the layer node $v_{3}$ (bus 9) from the ADN into the MG.

14): There is an ending node $v_{6}$ (bus 10) connected to the new transferred node $v_{2}$. Therefore, the ending node $v_{6}$ is also transferred from the ADN to the MG.

15): Set iteration $k=k+1=3$.

16): Update the MG with the further transferred loads from the ADN. The updated MG is shown in the circled area in Fig. 12 (c).

17): The operational constraints of the updated MG are satisfied by running power flow analysis. 
18): Check whether the required load curtailment $\Delta P_{L D}$ from the PSDC is satisfied in iteration $k=3$. In scenario 3, the required load curtailment from the PSDC is $\Delta P_{L D}=P_{k}-P_{\text {lim }, k}=32.02 \mathrm{MW}-19.2 \mathrm{MW}=12.82 \mathrm{MW}$. The capacity of current transferred loads is 16.1 MW in current iteration, which is contributed as load curtailment from the ADN side. Apparently, the required load curtailment from the PSDC is satisfied, i.e., Eq. (21) is satisfied.

19): Stop Algorithm 2 and output the capacity of final load transferring from the ADN to MG, which is 16.1 MW, as shown in the circled area in Fig. 12 (c).
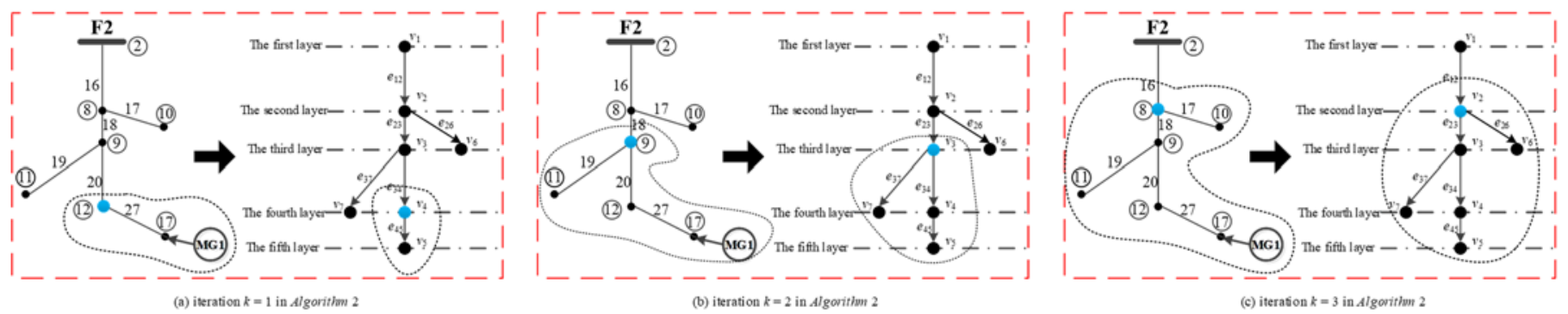

Fig. 12. Change of the MG containing updated transferred loads in electric feeder F2 in Case I.

The load transferring result in demand response scheme (as shown in Fig. 13) shows that the bus 9, 11, 8, 10 have been further transferred into the MG on the basis of load transferring scheme. Then the capacity of EVs load shedding from the EVCS is determined by running Problem II to maintain the reliable and secure operation of the MG. The results of the optimization problem II are shown in TABLE IV.

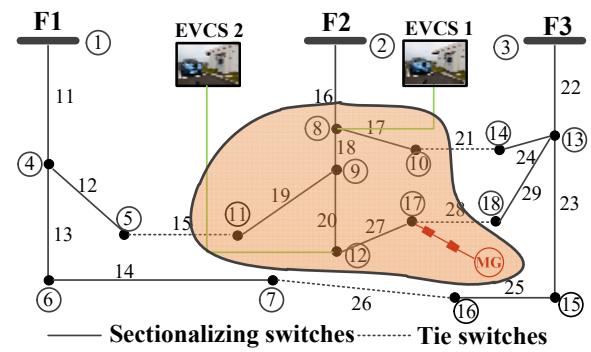

Fig. 13. The transferred loads in demand response scheme in Case $I$.

TABLE IV

The Results of Optimization PRoblem II In Demand Response Scheme of CASE I

\begin{tabular}{cccc}
\hline \hline Power Output from MGs (MVA) & Load Shedding from EVCS1 (MW) & Load Shedding from EVCS2 (MW) & Line Loss (MW) \\
\hline $13.0+\mathrm{j} 6.889$ & 3.1988 & 0 \\
\hline \hline
\end{tabular}

By utilizing the demand response scheme, the power flow of line $L_{19-20}$ is reduced to $16.53 \mathrm{MW}$, which is below its thermal limit.

The simulation results shown in TABLE IV suggest that the MG can operate reliably after the EVs load shedding of the EVCSs.

The simulation results in Scenario 3 demonstrate that the demand response scheme can alleviate overload in some critical lines of transmission network, while consider both the overload relief requirement of the transmission network and the EV users' comfort level. 
The results of overload relief of Case I in three different scenarios are shown in Fig. 14. It is shown that the ADN takes different operational schemes and adjusts its operational status (i.e., reconfiguring its network topology, transferring loads to MGs and shedding the EVs charging loads) to reduce the tie-line power between the transmission network and ADN under different overload situations of the transmission network. Then the overloads of line $L_{19-20}$ can be alleviated in the three scenarios.

It can be observed from Fig. 14 that with the decreasing of the thermal limits of $L_{19-20}$ in different scenarios of Case I (i.e., original value in scenario $1 \rightarrow 85 \%$ of its original value in scenario $2 \rightarrow 60 \%$ of its original value in scenario 3 ), the overload of the transmission network is getting more and more serious. Therefore, different operational schemes with different contributions of load curtailment from the ADN side, i.e., the active reconfiguration scheme, the load transferring scheme and the demand response scheme, are selected for the ADN to alleviate the overload in the transmission network.

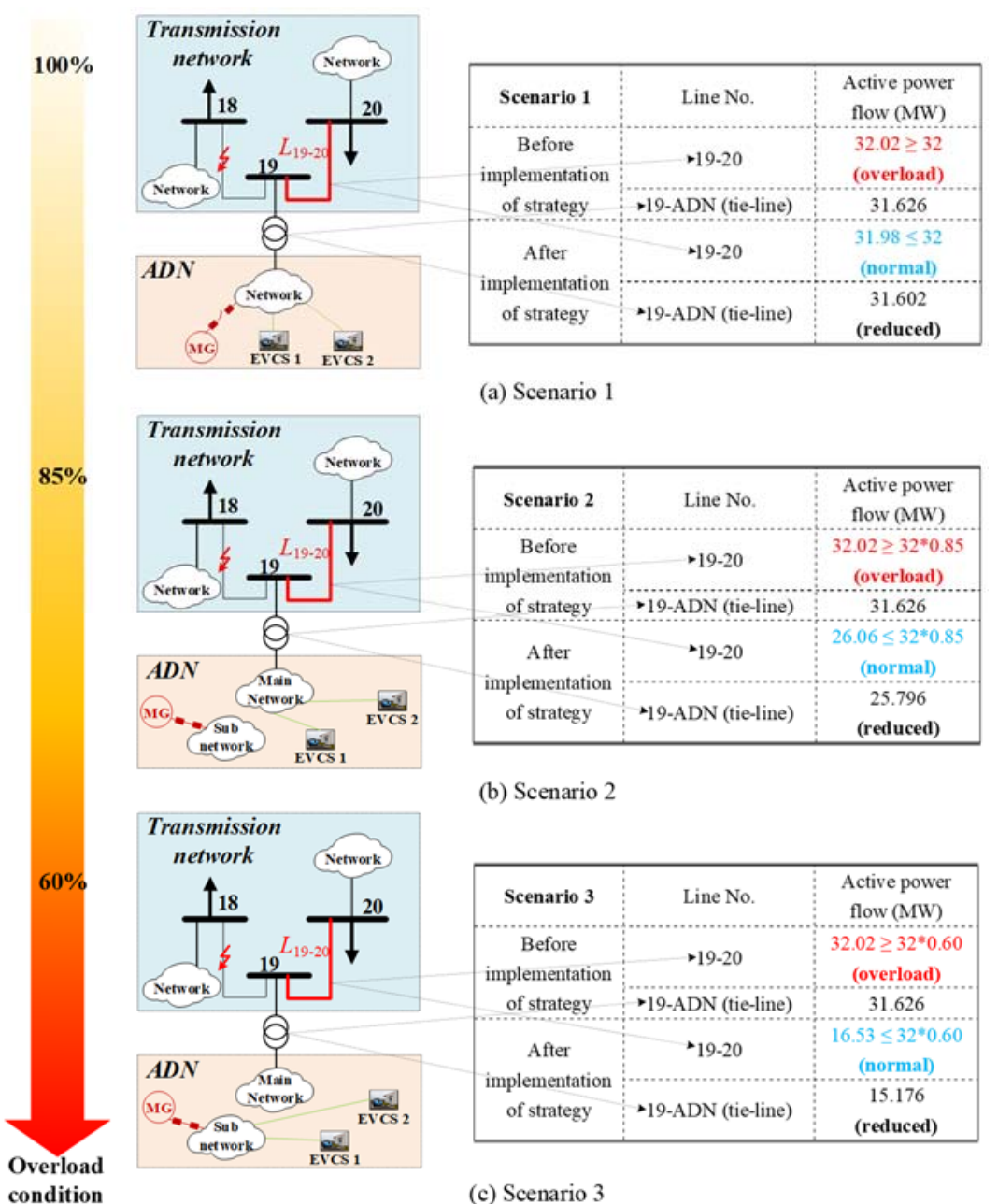

Fig. 14. The results of overload relief in three scenarios of Case $I$.

\section{B. Case II}

The 84-bus system of the Taiwan Power Company [41] is used as the second ADN case, as shown in Fig. 15. Three MGs are connected at bus 8,22 , and 40 . Three EVCSs are connected at bus 6,18 , and 34 . The maximum power output of the three MGs are 
set to be 1.5+j1.15MVA, 2.5+j1.92MVA, and 1.0+j0.77MVA. The maximum EV charging load of each EVCS of feeder A, C and $\mathrm{E}$ are set to be $1.5 \mathrm{MW}$ respectively in case II. The margin coefficient $k_{D}$ and $\alpha$ are set to be 1.2 and 0.95 respectively.

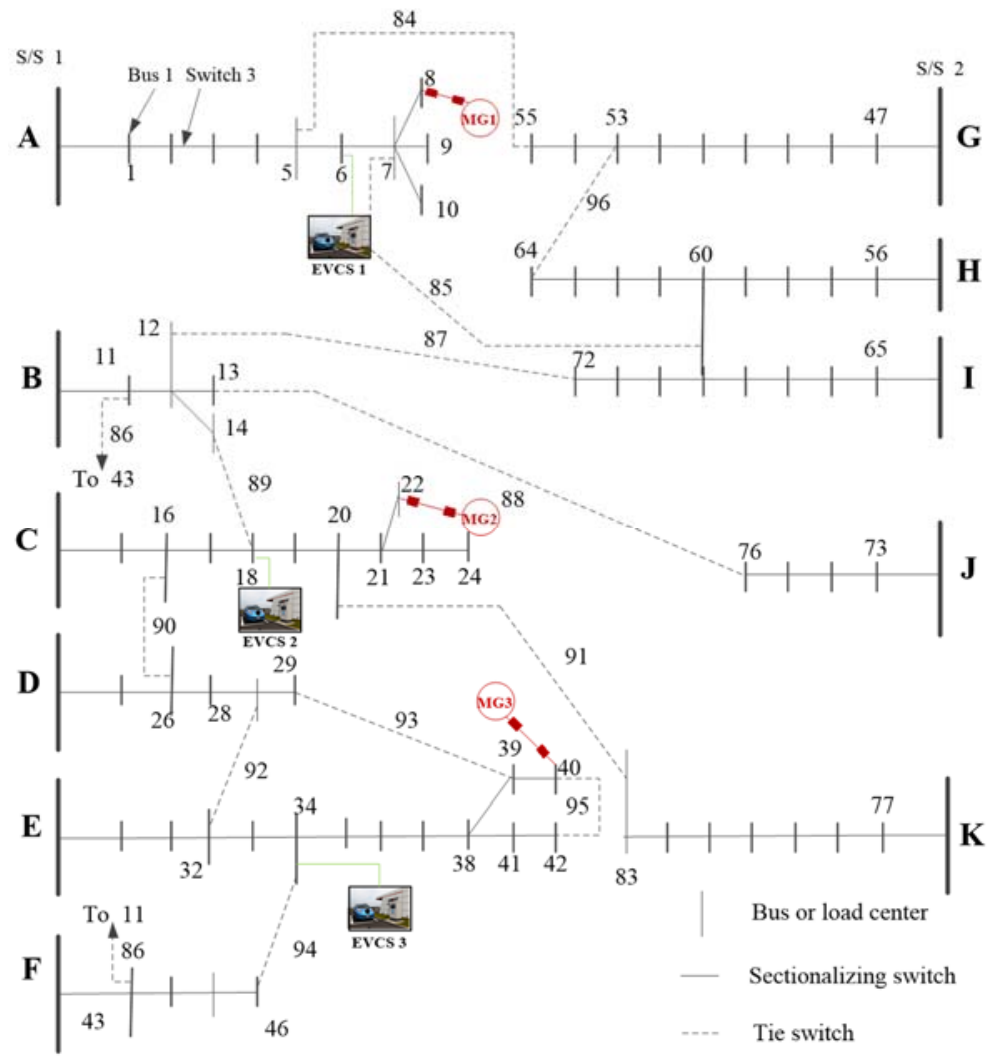

Fig. 15. Test ADN of Case II.

\section{1) Scenario 4: Simulation results for the active reconfiguration scheme}

The thermal limit of line $L_{19-20}$ is set to be $75 \%$ of its original value in Scenario 4 , which is $24 \mathrm{MW}$. When a fault occurs at $L_{18-19}$, the power flow of line $L_{19-20}$ is $24.36 \mathrm{MW}$ (obtained by running power flow of the transmission network), which violates its thermal limit (24MW). The $\Delta P_{L D}$ is $0.36 \mathrm{MW}^{5}$ and the $\Delta P_{e, l}$ is $0.5287 \mathrm{MW}$ (obtained through power flow calculation, as shown in TABLE V) in this scenario, which meets Eq. (1) and the active reconfiguration scheme is chosen.

TABLE V

FEEDER RECONFIGURATION RESULTS OF THE ADN IN SCENARIO 4

\begin{tabular}{cccc}
\hline \hline & Open Switches & Line Loss (MW) & Loss reduction (\%) \\
\hline Before Reconfiguration & $84,85,86,87,88,89,90,91,92,93,94,95,96$ & 1.3590 & - \\
After Reconfiguration & $4,33,37,52,55,86,87,88,89,90,91,92,95$ & 0.8303 & 38.9 \\
\hline \hline
\end{tabular}

The results of feeder reconfiguration of the ADN are shown in TABLE V. The power flow of line $L_{19-20}$ is $23.27 \mathrm{MW}$ after conducting the active reconfiguration scheme, which is below its thermal limit and the overload problem in $L_{19-20}$ is solved. The

${ }^{5} \Delta P_{L D}=P_{k}-P_{\text {lim }, k}=24.36 \mathrm{MW}-24 \mathrm{MW}=0.36 \mathrm{MW}$. 
results of overload relief in Scenario 4 of Case II are shown in Fig. 16 (a). It is shown that the overload of line $L_{19-20}$ can be alleviated by reconfiguring the network topology of the ADN in Scenario 4.

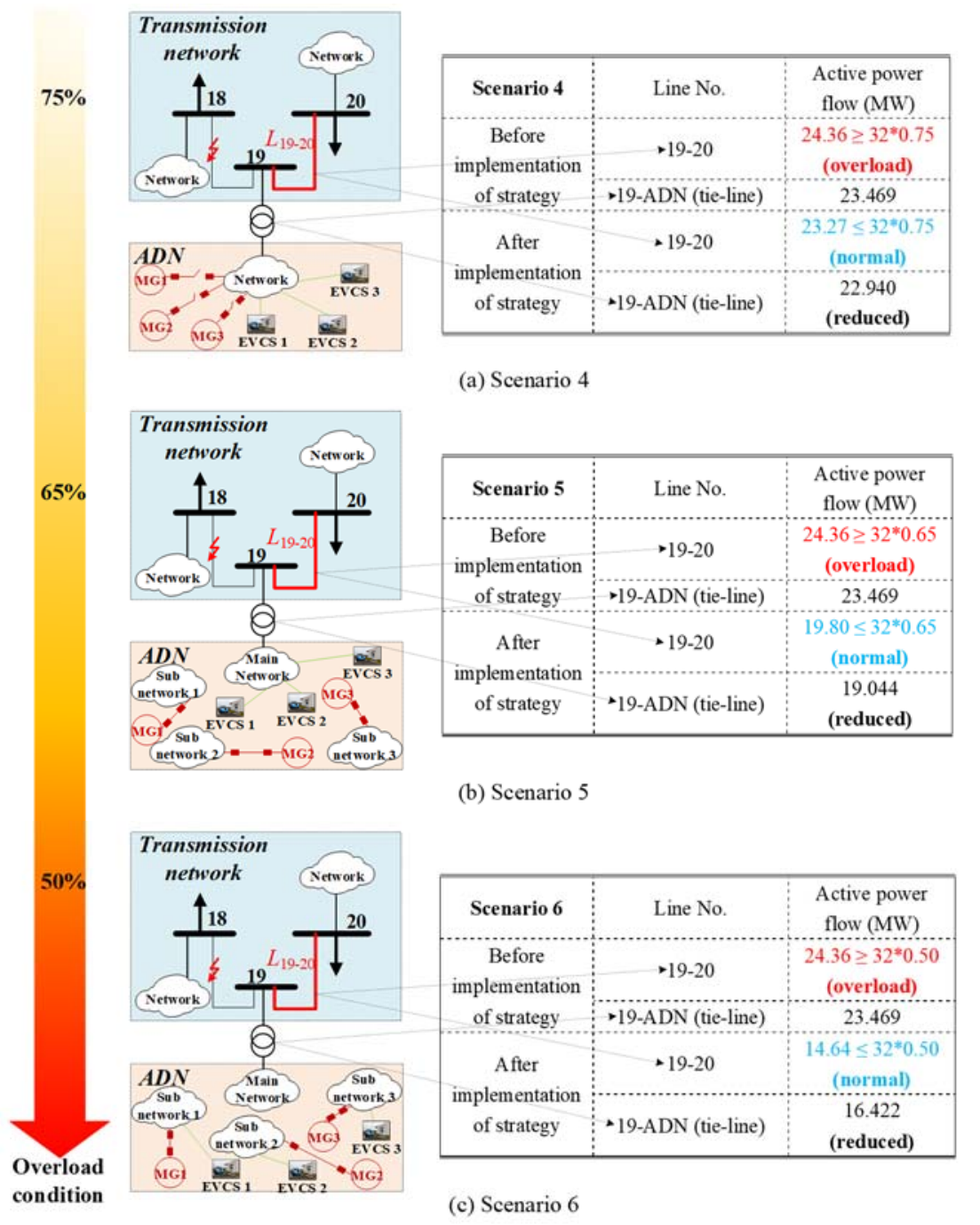

Fig. 16. The results of overload relief in three scenarios of Case II.

\section{2) Scenario 5: Simulation results for the load transferring scheme}

The thermal limit of line $L_{19-20}$ is set to be $65 \%$ of its original value in Scenario 5, which is $20.8 \mathrm{MW}$. If the ADMS choose the active reconfiguration scheme to alleviate the overload in transmission network in this scenario, the power flow of line $L_{19-20}$ would be 23.27MW. In this case, it violates the thermal limit of line $L_{19-20}$. Therefore, the active reconfiguration scheme fails to solve the overload problem and the load transferring scheme is chosen.

DFS of node-weight rooted tree is conducted to search the initial load transferring scheme firstly, and the final load transferring scheme is then decided after the feasibility check. The DFS algorithm for initial load transferring scheme in feeder A of the ADN is shown in Fig. 17. Firstly, the electric feeder A of the ADN is modelled as a nine-layer hierarchical rooted tree, as shown in the left 
side figure in Fig. 17. Then, the initial load transferring scheme can be obtained by implementing Algorithm 1. The specific steps are the same as the search steps in scenario 2 and the details are not presented in scenario 5 due to limited space. The initial load transferring scheme is then obtained, as shown in the circled area in Fig. 17. As highlighted in red color in Fig. 17, the initial load transferring scheme is isolated between node $v_{7}$ and $v_{8}$, i.e., between bus 6 and 7 of feeder A. The change of the rooted trees during the search process of the initial load transferring scheme in electric feeder A is shown in Fig. 18. It can be observed that the rooted tree is updated continuously until a subtree meeting PBC is found during the search process. The other initial load transferring schemes in feeder $\mathrm{C}$ and feeder $\mathrm{E}$ can also be determined by implementing the same search steps.

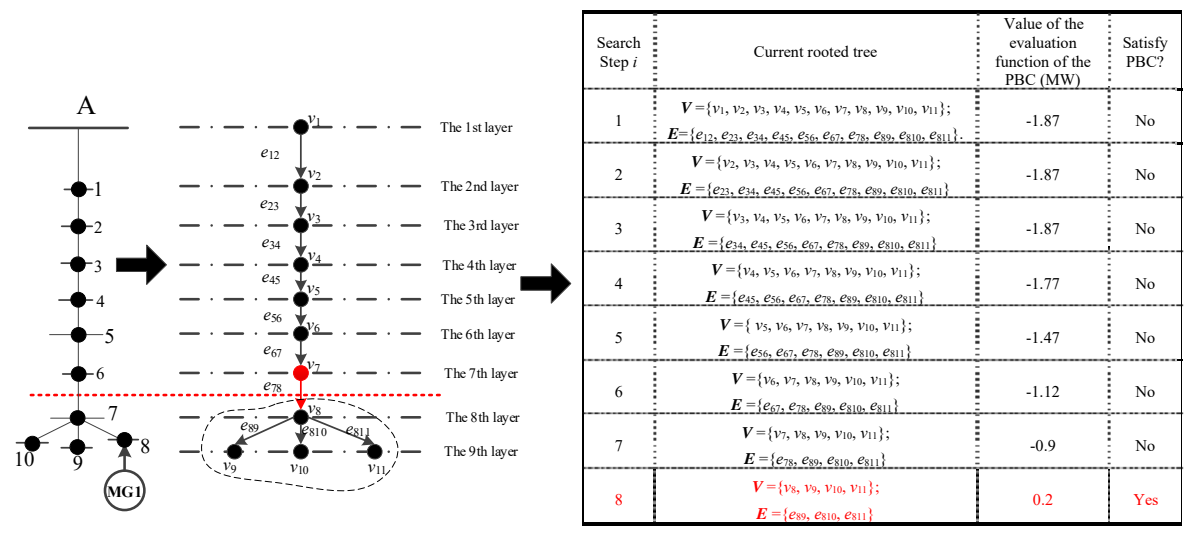

Fig. 17. DFS algorithm for initial load transferring scheme in feeder A of the ADN in Case II.

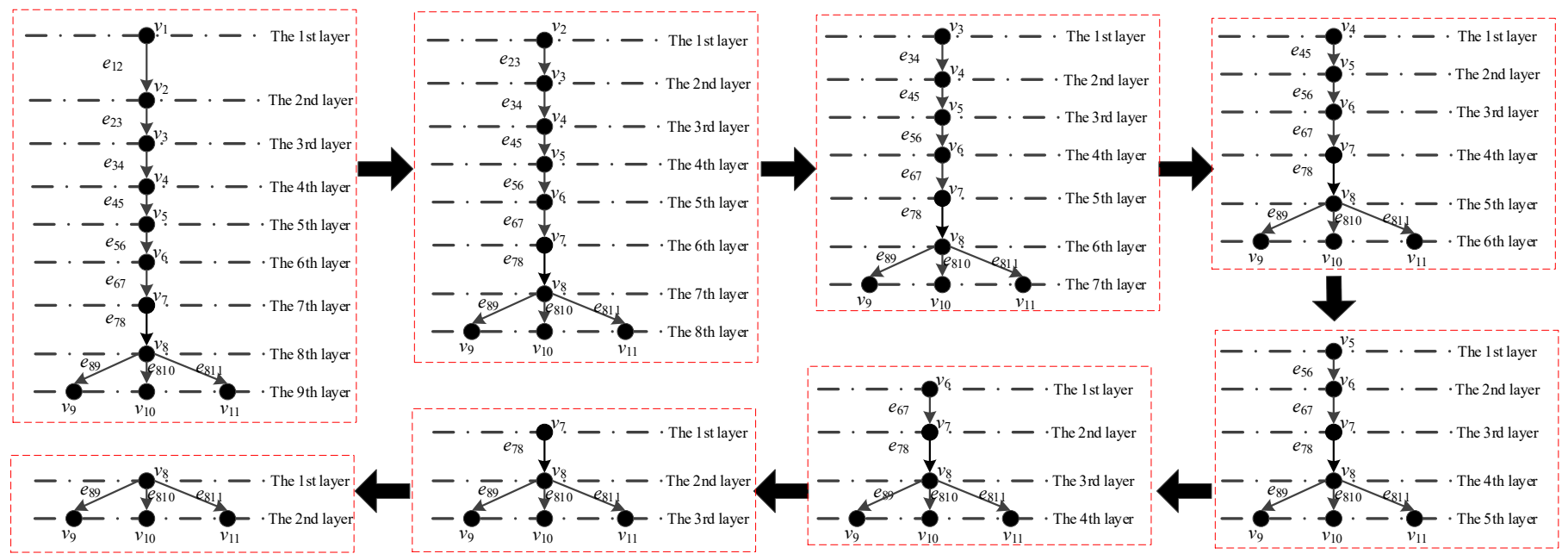

Fig. 18. Change of the rooted trees during the search process of the initial load transferring scheme in electric feeder A of the ADN in Case II.

Then, the feasibility of the initial load transferring is checked with the constraints from Eqs. (16) (19). After conducting the initial load transferring scheme, the power flow of line $L_{19-20}$ is reduced to $19.80 \mathrm{MW}$, which is below its thermal limit. In this scenario, the $\Delta P_{L D}$ is $3.56 \mathrm{MW}$ and the total capacity of load transferring from the ADN to MGs, i.e., $\sum P_{L i}$, is $3.88 \mathrm{MW}^{6}$, which meets the constraint (19), i.e. there is no load over-shedding from the ADN in the load transferring scheme. Other constraints are 
also met after the feasibility check. Therefore, the final load transferring scheme is determined, as depicted in Fig. 19. The operational results of the MGs containing transferred loads are shown in TABLE VI. The results of overload relief in Scenario 5 of Case II are shown in Fig. 16 (b). It is shown that the overload of line $L_{19-20}$ can be alleviated by transferring proper capacity of loads to the three MGs in Scenario 5.

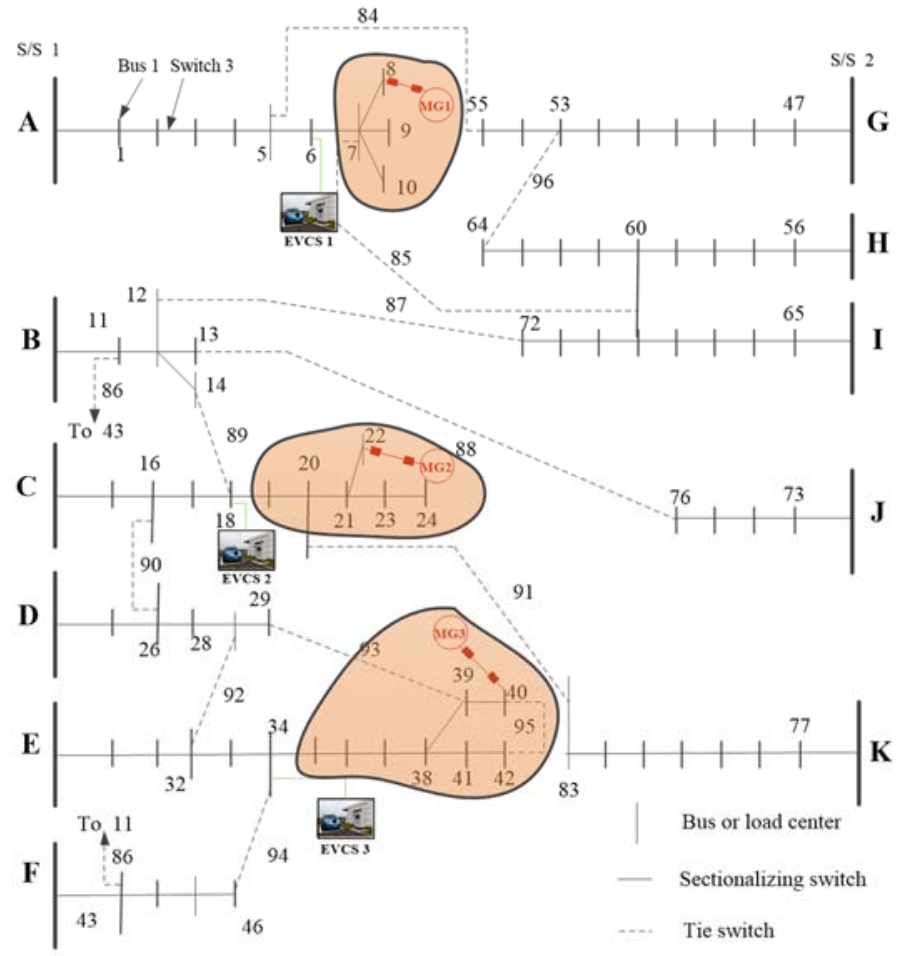

Fig. 19. The final load transferring scheme in Case II.

TABLE VI

RESULTS OF THE LOAD TRANSFERRING SCHEME IN CASE II

\begin{tabular}{cccc}
\hline \hline No. & Power Output from MGs (MVA) & Load Shedding from EVCS (MW) & Line Loss (MW) \\
\hline 1 & $1.3053+\mathrm{j} 1.0208$ & 0 & 0.0053 \\
2 & $2.0575+\mathrm{j} 1.7153$ & 0 & 0.0075 \\
3 & $0.5332+\mathrm{j} 0.3567$ & 0 & 0.0032 \\
\hline \hline
\end{tabular}

\section{3) Scenario 6: Simulation results for the demand response scheme}

The thermal limit of line $L_{19-20}$ is set to be $50 \%$ of its original value in Scenario 6, which is $16.0 \mathrm{MW}$. If the ADMS choose the load transferring scheme to alleviate the overload in transmission network in this scenario, the power flow of line $L_{19-20}$ would be 19.80MW (obtained in Scenario 5). In this case, it violates the thermal limit of line $L_{19-20}$. Therefore, the load transferring scheme fails to solve the overload problem and the demand response scheme is chosen.

Problem I is solved firstly to obtain the capacity of further load transferring from the ADN to the MG on the basis of load transferring scheme. It can be solved by implementing Algorithm 2. The specific steps are the same as the steps for implementing Algorithm 2 in scenario 3 and the details are not presented in scenario 6 due to limited space. The change of the MG containing 
updated transferred loads in electric feeder A in Case II is shown in Fig. 20. Algorithm 2 is stopped at iteration $k=2$ and the final transferred loads from the ADN to MG is shown in the circled area in in Fig. 20 (b).
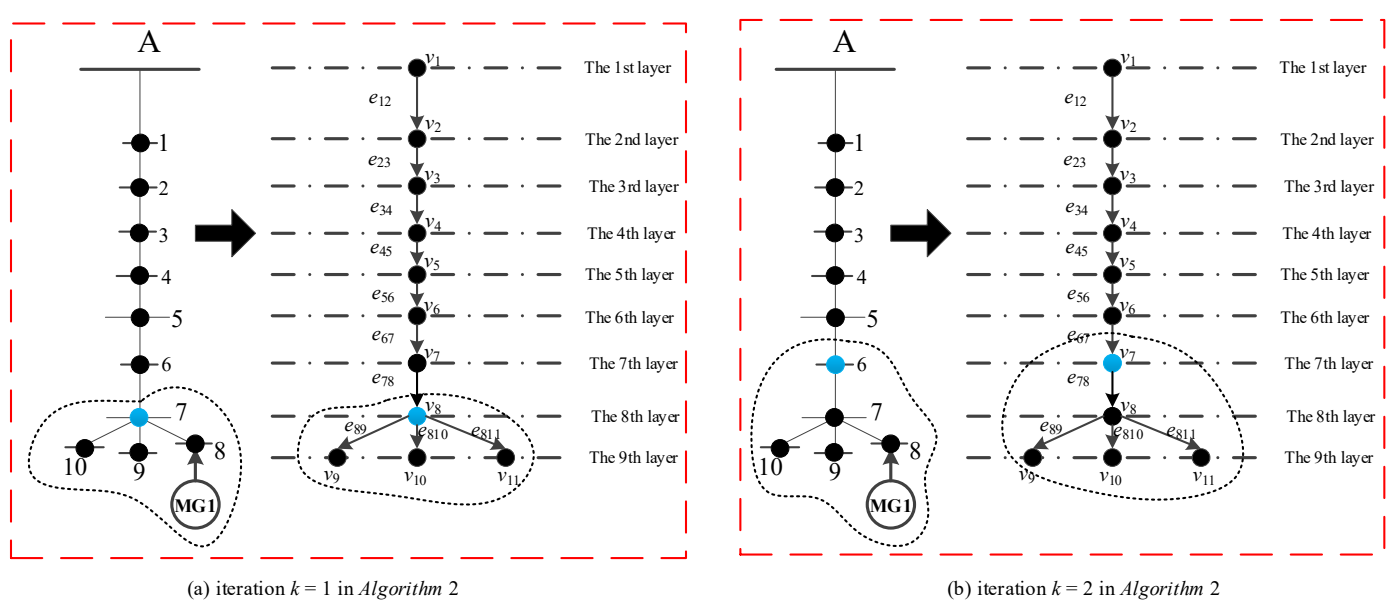

Fig. 20. Change of the MG containing updated transferred loads in electric feeder $A$ in Case II.

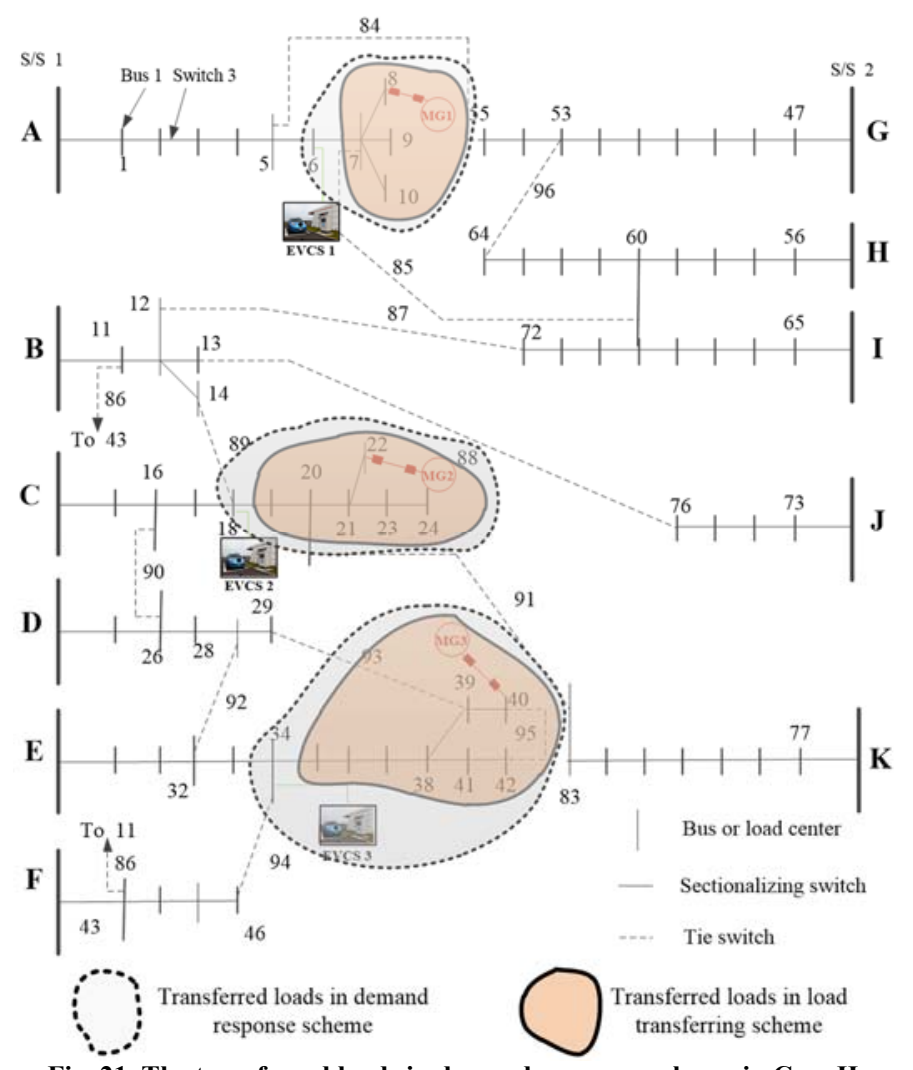

Fig. 21. The transferred loads in demand response scheme in Case II.

The result of the transferred loads from the ADN to the MGs in demand response scheme are depicted in Fig. 21. It shows that bus $6,18,34$ have been further transferred to the three MGs respectively on the basis of load transferring scheme. The further transferred loads to the three MGs in optimization problem I are the active power of bus $6(1.1 \mathrm{MW}), 18(0.7 \mathrm{MW}), 34(0.8 \mathrm{MW})$. 
Then the capacity of EVs load shedding from the EVCS are determined by solving the optimization problem II to maintain the reliable and secure operation of the MG. The results of the optimization problem II are shown in TABLE VII.

By utilizing the demand response scheme, the power flow of line $L_{19-20}$ is reduced to $14.64 \mathrm{MW}$, which is below its thermal limit (16.0MW). The simulation results shown in TABLE VII suggest that the MGs can operate reliably after the EVs load shedding of the EVCSs. The results of overload relief in Scenario 6 of Case II are shown in Fig. 16 (c). It is shown that the overload of line $L_{19-20}$ can be alleviated by transferring larger capacity of loads to the three MGs and shedding proper capacity of EV loads in Scenario 6.

TABLE VII

The Results of Optimization PRoblem II in Demand Response SCHEME of CASE II

\begin{tabular}{|c|c|c|c|}
\hline No. & Power Output from MGs (MVA) & Load Shedding from EVCS (MW) & Line Loss (MW) \\
\hline 1 & $1.5+\mathrm{j} 1.0673$ & 0.9073 & 0.0073 \\
\hline 2 & $2.5+\mathrm{j} 1.4619$ & 0.2594 & 0.0094 \\
\hline 3 & $1.0+\mathrm{j} 0.5351$ & 0.3351 & 0.0051 \\
\hline
\end{tabular}

It can be observed from Fig. 16 that with the decreasing of the thermal limits of $L_{19-20}$ in different scenarios of Case II (i.e., $75 \%$ of its original value in scenario $4 \rightarrow 65 \%$ of its original value in scenario $5 \rightarrow 50 \%$ of its original value in scenario 6 ), the overload of the transmission network is getting more and more serious. Therefore, different operational schemes with different contributions of load curtailment from the $\mathrm{ADN}$ side, i.e., the active reconfiguration scheme, the load transferring scheme and the demand response scheme, are selected for the $\mathrm{ADN}$ to alleviate the overload in the transmission network.

\section{Conclusions}

In this paper, a multi-level method to alleviate overload in transmission network using the capability from ADN is proposed. The following conclusions were derived based on the numerical studies:

1) The proposed multi-level method can alleviate the overload in transmission network using the capability from the ADN under the CTDM framework.

2) A proper operational scheme for the $\mathrm{ADN}$ (i.e., the active reconfiguration scheme, the load transferring scheme or the demand response scheme) can be determined according to the overload relief request from the transmission network.

3) The overload relief of the transmission network, the secure operation of the ADN, MGs and the customer comfort level of EVs users can be realized simultaneously with the proposed multi-level method.

4) It can also be concluded that the demand response scheme can contribute the largest capacity of load curtailment from the ADN side while the active reconfiguration scheme contributes the smallest capacity of load curtailment from the ADN side.

There are also limitations for the work carried out, and these are considered as the future research to be undertaken: 
1) The flexible capacity of RESs in the scope of the MGs are considered as the controllable power output of the MGs. The detailed formulation of RESs' participation in helping alleviate overload in the transmission network will be investigated in our future research work.

2) The flexible capacity of an ADN is small compared with the transmission network. However, the flexible capacity of individual ADNs can be aggregated to generate large capacity of flexibility. Therefore, how to use the flexibility from multiple ADNs for helping the transmission network with its operational issues is considered as the future research to be undertaken.

\section{ACKNOWLEDGEMENTS}

This work was financially supported by the National Key Research and Development Program of China (Grant no. 2017YFB0903300), the project of National Natural Science Foundation of China (Grant Nos. 51677124, and 51625702), and the Joint Fund Project of National Natural Science Foundation- State Grid Corporation (Grant no. U1766210).

\section{REFERENCES}

[1] H. S. Bidgoli and T. Van Cutsem, "Combined Local and Centralized Voltage Control in Active Distribution Networks," IEEE Trans. Power Syst., vol. 33, no. 2, pp. 1374-1384, 2018.

[2] H. Sun, Q. Guo, B. Zhang, Y. Guo, Z. Li, and J. Wang, "Master-Slave-Splitting Based Distributed Global Power Flow Method for Integrated Transmission and Distribution Analysis,” IEEE Trans. Smart Grid, vol. 6, no. 3, pp. 1484-1492, 2015.

[3] A. Kargarian and Y. Fu, "System of systems based security-constrained unit commitment incorporating active distribution grids,” IEEE Trans. Power Syst., vol. 29, no. 5, pp. 2489-2498, 2014.

[4] Z. Li, Q. Guo, H. Sun, and J. Wang, "Coordinated economic dispatch of coupled transmission and distribution systems using heterogeneous decomposition,” IEEE Trans. Power Syst., vol. 31, no. 6, pp. 4817-4830, 2016.

[5] Z. Li, Q. Guo, H. Sun, and J. Wang, "A new LMP-sensitivity-based heterogeneous decomposition for transmission and distribution coordinated economic dispatch,” IEEE Trans. Smart Grid, vol. 9, no. 2, pp. 931-941, 2018.

[6] H. Jia, W. Qi, Z. Liu, B. Wang, Y. Zeng, and T. Xu, "Hierarchical risk assessment of transmission system considering the influence of active distribution network,” IEEE Trans. Power Syst., vol. 30, no. 2, pp. 1084-1093, 2015.

[7] J. Liu, H. Cheng, P. Zeng, L. Yao, C. Shang, and Y. Tian, "Decentralized stochastic optimization based planning of integrated transmission and distribution networks with distributed generation penetration,” Appl. Energy, vol. 220, no. March, pp. 800813, 2018. 
[8] Z. S. Li, J. H. Wang, H. B. Sun, and Q. L. Guo, “Transmission Contingency Analysis Based on Integrated Transmission and Distribution Power Flow in Smart Grid,” IEEE Trans. Power Syst., vol. 30, no. 6, pp. 3356-3367, 2015.

[9] Z. Li, J. Wang, H. Sun, and Q. Guo, “Transmission Contingency Screening Considering Impacts of Distribution Grids," IEEE Trans. Power Syst., vol. 31, no. 2, pp. 1659-1660, 2016.

[10]L. Chen, H. Y. Li, S. Cox, and K. Bailey, “Ancillary Service for Transmission Systems by Tap Stagger Operation in Distribution Networks,” IEEE Trans. Power Deliv., vol. 31, no. 4, pp. 1701-1709, 2016.

[11]J. Villar, R. Bessa, and M. Matos, "Flexibility products and markets: Literature review,” Electr. Power Syst. Res., vol. 154, pp. $329-340,2018$.

[12] C. Liu, F. Chi, X. Jin, Y. Mu, H. Jia, and Y. Qi, “A multi-level control strategy for transmission congestion relief based on the capability from active distribution network," POWERCON 2014 - 2014 Int. Conf. Power Syst. Technol. Towar. Green, Effic. Smart Power Syst. Proc., no. Powercon, pp. 2000-2006, 2014.

[13] Y. Wang et al., "Pareto optimality-based multi-objective transmission planning considering transmission congestion," Electr. Power Syst. Res., vol. 78, no. 9, pp. 1619-1626, 2008.

[14]T. Duong, Y. Jiangang, and V. Truong, "A new method for secured optimal power flow under normal and network contingencies via optimal location of TCSC,” Int. J. Electr. Power Energy Syst., vol. 52, no. 1, pp. 68-80, 2013.

[15]N. Kirthika and S. Balamurugan, "A new dynamic control strategy for power transmission congestion management using series compensation,” Int. J. Electr. Power Energy Syst., vol. 77, pp. 271-279, 2016.

[16]Y. Mu, H. Jia, P. Zeng, X. Yu, and T. Xu, “A directional control method for interface flow considering static voltage stability,” Int. J. Electr. Power Energy Syst., vol. 64, pp. 176-184, 2015.

[17] S. R. SALKUTI, “Multi-Objective based Congestion Management using Generation Rescheduling and Load Shedding," IEEE Trans. Power Syst., vol. 32, no. 2, pp. 1-1, 2016.

[18]C. Venkaiah and D. M. Vinod Kumar, "Fuzzy adaptive bacterial foraging congestion management using sensitivity based optimal active power re-scheduling of generators,” Appl. Soft Comput. J., vol. 11, no. 8, pp. 4921-4930, 2011.

[19] S. Robak, J. MacHowski, and K. Gryszpanowicz, "Automatic Alleviation of Overloads in Transmission Network by Generation Curtailment,” IEEE Trans. Power Syst., vol. 33, no. 4, pp. 4424-4432, 2018.

[20] A. Ketabi and M. H. Fini, “An Underfrequency load shedding scheme for hybrid and Multiarea power systems,” IEEE Trans. Smart Grid, vol. 6, no. 1, pp. 82-91, 2015. 
[21]L. D. Arya and A. Koshti, "Anticipatory load shedding for line overload alleviation using Teaching learning based optimization (TLBO),” Int. J. Electr. Power Energy Syst., vol. 63, pp. 862-877, 2014.

[22] C.-M. Wang, P. Song, Y. Zhang, and W.-Q. Sun, "Flexible load shedding strategy considering real-time dynamic thermal line rating,” IET Gener. Transm. Distrib., vol. 7, no. 2, pp. 130-137, 2013.

[23] E. Hajipour, M. Bozorg, and M. Fotuhi-Firuzabad, "Stochastic Capacity Expansion Planning of Remote Microgrids with Wind Farms and Energy Storage,” IEEE Trans. Sustain. Energy, vol. 6, no. 2, pp. 491-498, 2015.

[24]H. Gao, J. Liu, L. Wang, and Z. Wei, “Decentralized Energy Management for Networked Microgrids in Future Distribution Systems,” IEEE Trans. Power Syst., vol. 33, no. 4, pp. 3599-3610, 2018.

[25]Y. Mu, J. Wu, N. Jenkins, H. Jia, and C. Wang, “A Spatial-Temporal model for grid impact analysis of plug-in electric vehicles," Appl. Energy, vol. 114, pp. 456-465, 2014.

[26]F. F. Wu, K. Moslehi, A. Bose, “Power system control centers: Past, present, and future,” Proceedings of the IEEE, vol. 93, no. 11, pp. 1890-1908, 2005.

[27] S. Golshannavaz, S. Afsharnia, and F. Aminifar, "Smart distribution grid: Optimal day-ahead scheduling with reconfigurable topology," IEEE Trans. Smart Grid, vol. 5, no. 5, pp. 2402-2411, 2014.

[28]B. Amanulla, S. Chakrabarti, and S. N. Singh, "Reconfiguration of power distribution systems considering reliability and power loss,” IEEE Trans. Power Deliv., vol. 27, no. 2, pp. 918-926, 2012.

[29] W. C. Wu and M. S. Tsai, “Application of enhanced integer coded particle swarm optimization for distribution system feeder reconfiguration,” IEEE Trans. Power Syst., vol. 26, no. 3, pp. 1591-1599, 2011.

[30] J. Olamaei, T. Niknam, and G. Gharehpetian, “Application of particle swarm optimization for distribution feeder reconfiguration considering distributed generators,” Appl. Math. Comput., vol. 201, no. 1-2, pp. 575-586, 2008.

[31]C. M. Chan, C. N. Lu, and Y. L. Lo, “Optimal Use of Existing Distribution Feeders to Accommodate Transportation Electrification,” IEEE Trans. Intell. Transp. Syst., vol. 16, no. 4, pp. 1941-1950, 2015.

[32] T. Niknam, “An efficient hybrid evolutionary algorithm based on PSO and HBMO algorithms for multi-objective Distribution Feeder Reconfiguration,” Energy Convers. Manag., vol. 50, no. 8, pp. 2074-2082, 2009.

[33]X. Jin, Y. Mu, H. Jia, J. Wu, X. Xu, and X. Yu, “Optimal day-ahead scheduling of integrated urban energy systems,” Appl. Energy, vol. 180, pp. 1-13, 2016.

[34] T. Niknam, A. Kavousifard, and J. Aghaei, "Scenario-based multiobjective distribution feeder reconfiguration considering wind power using adaptive modified particle swarm optimisation,” IET Renew. Power Gener., vol. 6, no. 4, p. $236,2012$. 
[35] R. A. Jabr, R. Singh, and B. C. Pal, “Minimum loss network reconfiguration using mixed-integer convex programming,” IEEE Trans. Power Syst., vol. 27, no. 2, pp. 1106-1115, 2012.

[36] Y. Y. Hong and S. Y. Ho, "Determination of network configuration considering multi-objective in distribution systems using genetic algorithms,” IEEE Trans. Power Syst., vol. 20, no. 2, pp. 1062-1069, 2005.

[37] X. Jin, T. Jiang, Y. Mu, C. Long, X. Li, H. Jia, and Z. Li, "Scheduling distributed energy resources and smart buildings of a microgrid via multi-time scale and model predictive control method,” IET Renew. Power Gener., vol. 13, no. 6, pp. 816-833, 2019.

[38]E. Bompard, T. Huang, and L. Yang, "Market equilibrium under incomplete and imperfect information in bilateral electricity markets," IEEE Trans. Power Syst., vol. 26, no. 3, pp. 1231-1240, 2011.

[39] R. D. Zimmerman, C. E. Murillo-Sánchez, and R. J. Thomas, “MATPOWER: Steady-state operations, planning, and analysis tools for power systems research and education,” IEEE Trans. Power Syst., vol. 26, no. 1, pp. 12-19, 2011.

[40]K. Nara, A. Shiose, M. Kitagawa, "Implementation of genetic algorithm for distribution systems loss minimum re-configuration,” IEEE Trans. Power Syst., vol. 7, no. 3, pp. 1044-1051, 1992.

[41]J. Chiou, C. Chang, C. Su, and S. Member, "Variable Scaling Hybrid Differential Evolution for Solving Network Reconfiguration of Distribution Systems,” IEEE Trans. Power Syst., vol. 20, no. 2, pp. 668-674, 2005. 